BNL-77185-2006-BC

\title{
Spin Polarized Photoelectron Spectroscopy as a Probe of Magnetic Systems
}

\author{
P.D. Johnson and G. Güntherodt
}

To be published in "The Handbook of Magnetism and Advanced Magnetic Materials”

October 2006

\author{
Condensed Matter Physics \& Materials Science Department \\ Brookhaven National Laboratory \\ P.O. Box 5000 \\ Upton, NY 11973-5000 \\ www.bnl.gov
}

\begin{abstract}
Notice: This manuscript has been authored by employees of Brookhaven Science Associates, LLC under Contract No. DE-AC02-98CH10886 with the U.S. Department of Energy. The publisher by accepting the manuscript for publication acknowledges that the United States Government retains a non-exclusive, paid-up, irrevocable, world-wide license to publish or reproduce the published form of this manuscript, or allow others to do so, for United States Government purposes.
\end{abstract}

This preprint is intended for publication in a journal or proceedings. Since changes may be made before publication, it may not be cited or reproduced without the author's permission. 


\section{DISCLAIMER}

This report was prepared as an account of work sponsored by an agency of the United States Government. Neither the United States Government nor any agency thereof, nor any of their employees, nor any of their contractors, subcontractors, or their employees, makes any warranty, express or implied, or assumes any legal liability or responsibility for the accuracy, completeness, or any third party's use or the results of such use of any information, apparatus, product, or process disclosed, or represents that its use would not infringe privately owned rights. Reference herein to any specific commercial product, process, or service by trade name, trademark, manufacturer, or otherwise, does not necessarily constitute or imply its endorsement, recommendation, or favoring by the United States Government or any agency thereof or its contractors or subcontractors. The views and opinions of authors expressed herein do not necessarily state or reflect those of the United States Government or any agency thereof. 


\title{
Spin-Polarized Photoelectron Spectroscopy as a probe of Magnetic
}

\section{Systems}

\section{P.D. Johnson ${ }^{1}$ and G. Güntherodt ${ }^{2}$}

1. Condensed Matter Physics and Materials Science Department Brookhaven National Laboratory, Upton, New York, 11973

2. Physics Institute IIA, RWTH Aachen University, 52056 Aachen, Germany

\begin{abstract}
Spin-polarized photoelectron spectroscopy has developed into a versatile tool for the study of surface and thin film magnetism. In this chapter, we examine the methodology of the technique and its recent application to a number of different problems. We first examine the photoemission process itself followed by a detailed review of spinpolarization measurement techniques and the related experimental requirements. We review studies of spin polarized surface states, interface states and quantum well states followed by studies of the technologically important oxide systems including halfmetallic transition metal oxides, ferromagnet/oxide interfaces and the antiferromagnetic cuprates that exhibit high Tc Superconductivity. We also discuss the application of high-resolution photoemission with spin resolving capabilities to the study of spin dependent self energy effects.
\end{abstract}




\section{Introduction}

Driven by the requirements for new information storage technologies and the potential of "spintronics" the last two decades have seen a rapid increase in research devoted to studies of low dimensional magnetic systems. New technologies such as molecular beam epitaxy, previously developed in the semiconductor industry, are now being applied to the development of new magnetic materials with unique properties. The understanding of the properties of these two-dimensional (2D) systems has provided a number of exciting challenges for the scientific community, both experimental and theoretical.

On the experimental side a whole range of electron spectroscopies previously developed for the study of metallic surfaces have been 'spin-sensitized' through the addition of spin polarimeters. Electron-based techniques are particularly suited to the study of surfaces and thin films because the strong Coulomb interaction between electrons results in a relatively short mean free path and associated probing depth. In this chapter we examine in detail the recent developments of one such technique, spin polarized photoemission. Photoemission itself has already seen widespread application to the study of the electronic structure of a range of different materials (Kevan 1992, Hüfner, 2003). The spin-polarized counterpart has a history spanning a period in time that is almost as long as the 'modern' era of photoemission. However, it has taken several years for the complete angle-resolved, spin-resolved photoemission experiment to be developed to the point where it provides spectra with a signal-to-noise ratio comparable to that of its non-spin-resolved counterpart. 
Several reviews of spin-polarized photoelectron spectroscopy (SPPES) have already appeared in the literature (Feder 1985, Kisker 1987, Johnson 1997). The present review represents an attempt to provide a perspective on the rapid developments in the last ten years. We first examine the physics of the photoemission process itself with particular reference to the excitation of spin-polarized electrons. The experimental aspects including the different spin polarimeters that are currently available and the unique requirements of a polarimetry measurement are discussed. We then examine a whole range of recent experiments. We discuss recent studies of magnetic surface states observed on non-magnetic materials, spin polarized quantum well states that develop in nonmagnetic thin films deposited on ferromagnetic substrates, the measurement of spindependent self energy effects and finally studies of a range of magnetic oxides including both ferromagnetic and antiferromagnetic materials and ferromagnet/oxide interfaces. The properties of the quantum well states observed in thin films are particularly relevant to the discussion of the giant magneto-resistance (GMR) property observed in magnetic multilayers and studies of the oxides are relevant to the development of new capabilities in spintronics.

\section{The Photoemission Process}

In studies of condensed matter systems, photoemission represents the excitation of an electron from an initial state below the Fermi level to a final state above the vacuum level. As illustrated in figure 1, the initial state may fall within the delocalized valence bands or it may represent a more localized core level. In the absence of spin-orbit coupling, it can be shown for linearly polarized incident light that the non-relativistic 
Schrödinger equation, with the momentum operator $\boldsymbol{p}$ replaced by $\boldsymbol{\underline { p }}-(\mathrm{e} / \mathrm{c}) \underline{\boldsymbol{A}}$, represents an adequate description of the spin-conserving transitions. Here $\underline{A}=\underline{A_{0}} e^{i \omega t}$ is the vector potential of the electromagnetic field. From Fermi's Golden rule, the differential cross section $d \sigma / d \Omega$ for excitation from some initial state $\left|\psi_{i}\right\rangle$ to some final state $\left|\psi_{f}\right\rangle$ is given by

$$
\frac{d \sigma}{d \Omega}\left(E_{f}, \hbar \omega, k_{f}, \underline{A}\right) \propto \sqrt{E_{f}} \sum_{i}\left|\left\langle\psi_{f}|(\underline{A} \cdot \underline{p}+\underline{p} \cdot \underline{A})| \psi_{i}\right\rangle\right|^{2} \delta\left(E_{i}-E_{f}-\hbar \omega\right)
$$

where the $\delta$ function describes the energy conservation of the process. Measuring the kinetic energy of the electron in the final state $E_{f}$ and knowing the incident photon energy, $\hbar \omega$, the experimentalist can trace back to the binding energy of the electron in the initial state $E_{i}$.

Neglecting the diamagnetic term $|\mathrm{A}|^{2}$ which is always small and noting that $\nabla \cdot \underline{A}$ is non-zero only in the surface region, equation (1) is usually reduced to the simpler form

$$
\frac{d \sigma}{d \Omega} \propto \sqrt{E_{f}} \sum_{i}\left|\left\langle\psi_{f}|(\underline{A} \cdot \underline{p})| \psi_{i}\right\rangle\right|^{2} \delta\left(E_{i}-E_{f}-\hbar \omega\right)
$$

The matrix element introduces selection rules which can be exploited to determine the symmetry of the initial state. In the non-relativistic limit, excitation by linearly polarized light between one electron states of the form $\left|n l m_{l}\right\rangle$ is restricted to transitions such that $\Delta l= \pm 1$ and $\Delta m_{l}=0$. In the event that the incident light is circularly polarized, the second selection rule becomes $\Delta m_{l}= \pm 1$ dependent on the handedness of the polarization. In the fully relativistic treatment with spin-orbit coupling included, the selection rules become $\Delta j=0, \pm 1$ and $\Delta m_{j}= \pm 1$ with $j$ and $m_{j}$ now referring to the total angular momentum. 
A more complete description of the photoemission process will allow for the many-body response of the system to the excitation. Here the $\delta$ function of equation (2) is replaced by the single particle spectral function of the hole state $A(\underline{k}, \omega)$ such that

$$
A(\underline{k}, \omega)=\frac{1}{\pi} \frac{\Sigma_{2}(\underline{k}, \omega)}{\left[\omega-\varepsilon_{k}-\Sigma_{1}(\underline{k}, \omega)\right]^{2}+\left[\Sigma_{2}(\underline{k}, \omega)\right]^{2}}
$$

where the real component of the self energy, $\Sigma_{1}(\underline{k}, \omega)$, gives a shift in energy and associated mass enhancement and the imaginary component of the self energy, $\Sigma_{2}(\underline{k}, \omega)$, gives the lifetime broadening. Note that $\omega$ in equation (3) represents the initial state energy $E_{i}$ and $\varepsilon_{k}$ represents the bare band dispersion in the absence of any many-body interactions. In ferromagnetic systems, because of the spin polarization of the electronic structure, we may anticipate a spin-dependence in the response of the system.

Extending the technique and measuring the photoemitted current at some emission angle $\theta$ it becomes possible to map the dispersion of the different initial-state bands. As illustrated in figure 2, at a well defined $k_{\|}$, the process represents a direct transition between two bands of the same spin. In the solid state environment, momentum conservation is maintained through the mediation of the crystal momentum giving

$$
k_{f}=k_{i}+G
$$

where $k_{i}$ and $k_{f}$ are the wavevectors associated with the initial and final states and $G$ represents a suitable lattice vector. If $E_{k i n}$ represents the kinetic energy in the final state then to within a reciprocal lattice vector the parallel momentum $k_{\|}$of the photoelectron given by

$$
k_{\|}=\left(\frac{2 m}{\hbar^{2}}\right)^{1 / 2} E_{k i n}^{1 / 2} \sin \theta
$$


and is conserved on crossing from the solid into the vacuum. Thus a measurement of this component in the vacuum supplies a good measure of the parallel component of the momentum in the solid. The perpendicular component is ill-defined due to the breaking of translational symmetry perpendicular to the surface plane.

\section{The experimental methodolgy}

As in any electron spectroscopy, spin-polarized photoemission requires the use of an electron spectrometer. These instruments can take many different forms although, as illustrated in figure 3, the hemispherical analyzer represents the most commonly used instrument in SPES at the present time. A more complete discussion of the properties of such an analyser can be found elsewhere (e.g. Roy and Carette 1977). Here we note that in such an instrument the kinetic energy of the electron is measured by retarding electrostatic fields applied between the two hemispheres and the momentum is selected by defining a small angle of collection. As also illustrated in figure 3, some form of electrostatic lens is required to couple this analyser to whichever spin polarimeter is selected for the experiment.

An overview of the practicalities of coupling spin polarimeters to electron spectrometers has been given by Pierce et al (1988). In particular, these authors emphasize the requirement for optimizing the experiment by matching the phase space or acceptances of the source, the electron spectrometer and finally of the spin polarimeter itself. At any point on the pathway of the electron beam the Helmholz-Lagrange law states that there will be a conservation of the product $E A \Omega$, where $E$ is the energy, $\Omega$ the solid angle and $A$ the cross sectional area. In the optimum configuration this product will 
be matched to the acceptance phase space of the polarimeter. Pierce et al (1988) have tabulated the latter for a number of different spin polarimeters.

\subsection{Measuring the spin polarization}

The two principal means of measuring spin polarization involve either the use of spin dependent scattering via the spin-orbit interaction with a heavy atom or the use of an exchange interaction with a ferromagnetic material. We briefly examine these two different approaches. Much of the pioneering work in spin-polarized photoemission was carried out with spin polarimeters of the high-energy Mott-scattering type (Kisker et al 1982). Here the electrons to be analyzed are scattered off gold atoms at energies typically of the order of $100 \mathrm{keV}$. Spin-orbit coupling of the electron in the potential of the gold atom leads to an asymmetry in the scattering, left and right, dependent on the spin of the electron. Because of the high energies involved in the earlier designs, Mott polarimeters tended to be large. However, there have recently been a number of successful modifications that have allowed the polarimeter to be scaled down in size. Figure 4 shows the configuration used in one such device as described by Huang et al (2002). After initially passing through a deceleration lens the incident beam of electrons is accelerated onto the gold foil at an energy of $20 \mathrm{keV}$. As in the earlier designs (Kisker $e t$ al 1982), those electrons elastically scattered through $120^{\circ}$ are detected by two symmetrically opposite channeltrons. Retarding fields in front of the latter collectors

remove any electrons that have undergone inelastic losses. The spin polarization $P$ of the incident electron beam is given by

$$
P=\frac{1}{S} \frac{I_{A}-I_{B}}{I_{A}+I_{B}}
$$


where $S$, the Sherman function of the device, is a measure of its ability to distinguish different spins and $I_{A}$ and $I_{B}$ represent the intensities measured in opposite channels. Mott polarimeters of this type are now sufficiently small that they can in principle be readily moved inside a vacuum system and, therefore, be used for angle-resolved polarization measurements. The figure of merit (FOM) used in comparing different polarimeters is defined as

$$
F O M=S^{2} \frac{I}{I_{0}}
$$

where again $S$ is the Sherman function, $I$ is the sum of the current collected by the two opposite detectors and $I_{0}$ is the incident beam current (Kessler 1985). The FOM for the traditional Mott polarimeter is typically $10^{-4}$; that of the compact low-energy Mott device was initially of the order of $2 \times 10^{-5}$ (Tang et al 1988). However through the use of large area electron detectors and careful optimization of the electron optics the FOM of the compact devices has gradually been improved. Burnett et al. reported an FOM of approximately $1.6 \times 10^{-4}$. More recently Huang et al. (2002) have refined the electron optics further to achieve an FOM of $\sim 2 \times 10^{-4}$. The advantage of the Mott polarimeters, both large and small, is that they are relatively easy to operate in a reproducible fashion.

Originally designed for use in secondary electron microscopy with polarization analysis (SEMPA) studies (Unguris et al 1986, Scheinfein et al 1989), low energy diffuse scattering from polycrystalline gold films has also proved an excellent device for energyand angle-resolved spin-polarized photoemission studies (Johnson et al 1992). Rather than the high energies, characteristic of the Mott devices, the electrons to be spin analysed are now scattered from a polycrystalline gold surface at the much lower energy of $150 \mathrm{eV}$, an energy at which the Sherman function of gold exhibits a local maximum. 
The back-scattered electrons pass through retarding grids to remove low-energy secondary electrons, and are then collected by four discrete anodes allowing a measurement of two components of the polarization to be made in parallel. This type of detector has an FOM of the order of $1.0 \times 10^{-4}$ (Unguris et al 1986), i.e. comparable to the traditional Mott detector.

If the scattering surface is a single crystal rather than polycrystalline it is possible to use spin-dependent diffraction to measure the polarization. The spin asymmetry in the scattering again reflects the spin-orbit interaction. Detectors of this type include a lowenergy detector, which relies on spin-orbit effects in the diffraction of the electrons from a single-crystal tungsten (001) surface. (Kirschner and Feder, 1979) The intensities of the symmetrically opposite $(2 ; 0)$ and $(\overline{2} ; 0)$ diffracted beams are measured with the electrons incident at an energy of $100 \mathrm{eV}$. The authors report an FOM for this detector of $1.6 \times 10^{-4}$.

Spin polarimeters based on an exchange interaction fall into two categories, those employing reflection and those employing transmission. In practice only the reflection technique has been used in working spin polarimeters to date. A detector based on lowenergy reflection from a ferromagnetic film has been described by Tillman et al (1989). Here the scattering surface is a $400 \AA$ thick $\mathrm{Fe}(001)$ film grown on an $\mathrm{Ag}(001)$ substrate. Reflected intensities are measured when the electrons to be analysed are incident on a magnetized iron surface at an energy of approximately $10.0 \mathrm{eV}$. The asymmetry in the scattering, $A$, in such a detector is given by

$$
A=\frac{1}{P} \frac{I^{\uparrow \uparrow}-I^{\uparrow \downarrow}}{I^{\uparrow \uparrow}+I^{\uparrow \downarrow}}
$$


where $P$ is the spin polarization in the incident beam and $I^{\uparrow \uparrow}$ and $I^{\uparrow \downarrow}$ are the scattered intensities obtained when the target and primary beam magnetic moments are parallel and anti-parallel, respectively. The FOM of such a device is then given by

$$
F O M=A^{2} \frac{I_{R}}{I_{P}}
$$

where $I_{R}$ and $I_{P}$ are the reflected and primary beam currents, respectively. One difficulty with an instrument of this type is that with the reflecting or analyzing surface being effectively another 'sample', two samples have to be successfully prepared and magnetized. However, at $10.6 \mathrm{eV}$ analyzing energy, the authors report a value of 0.21 for $A$ and an FOM of $3.5 \times 10^{-3}$.

More recently the spin dependent reflectivity of electrons from Fe single crystal films, 2-8 monolayers thick, grown on a W(110) substrate has been studied by Zdyb and Bauer (Zdyb and Bauer, 2002). These authors analyzed their data in terms of the quantum well structure associated with the thin films, that we discuss in detail later. The authors went on to point out the importance of considerations of the quantum well structure in the design of future spin polarimeters. In a later study, Graf et al extended these ideas to studies of both Fe and Co thin films grown on W(110) (Graf et al., 2005). Again by measuring the spin dependent reflectivities as a function of film thickness the authors determined that a five monolayer thick film of $\mathrm{Co} / \mathrm{W}(110)$ represented the optimum configuration for a spin polarimeter. They further indicated that the FOM of such a device would be of the order of 0.02 , some two orders of magnitude higher than that achieved in the miniature Mott detectors.

Turning to transmission, Schönhense and Siegmann (1993) have suggested the possibility of using the spin-dependent transmission through ferromagnetic thin films as a 
means of detecting spin polarization. That such a spin dependence in transmission exists is evident in that the low-energy secondary electron cascade in all electron spectroscopies is known to display an enhanced spin polarization. The latter polarization which is typically larger than would be expected simply on the basis of the bulk valence band polarization reflects the spin-dependent mean free paths of the transmitted electrons. Schönhense and Siegmann examined a number of experiments in detail. As shown in figure 5 they find that the total scattering cross section $\sigma(\mathrm{E})=1 / \lambda$ is well described by the expression

$$
\sigma=\sigma_{0}+\sigma_{\mathrm{d}}(5-\mathrm{n})
$$

where $\sigma_{\mathrm{d}}$ accounts for scattering into the d-holes and $\sigma_{0}$ is a constant accounting for other inelastic scattering events. They define a transport polarization $\alpha$ such that an electron beam with small initial polarization $P_{0}$ will acquire a polarization after travelling a distance $x$ such that

$$
P(x)=P_{0}+\alpha(\mathrm{x})
$$

The transport polarization $\alpha$ is given by

$$
\left.\alpha=\frac{\exp \left(\sigma^{-}-\sigma^{+}\right) x-1}{\exp \left(\sigma^{-}-\sigma^{+}\right) x+1}\right]
$$

with $\sigma^{( \pm)}$representing the spin-dependent cross sections. In their picture the spin polarization is, therefore, dominated by the inelastic scattering involving unoccupied states above the Fermi level.

Schönhense and Siegmann note that a spin polarimeter based on the use of spin dependent transmission will have an FOM given by $\alpha^{2} I$ where $I=e^{-\alpha x}$ represents the total spin-integrated transmission through the film and $\sigma$ is the spin-averaged total cross 
section. On the basis of their experimental observations they show that the FOM for an iron film will peak at approximately $7 \times 10^{-2}$ for film thicknesses of approximately $1.0 \mathrm{~nm}$. For Co they predict an FOM of $6 \times 10^{-2}$ for a film thickness of approximately $1.5 \mathrm{~nm}$. These ideas have been given some support in studies of the spin-dependent transmission through a free standing Co film (Lassailly et al 1994, van der Sluijs et al 1994). The authors of the latter study found that at low energies, close to the surface vacuum level, the transmission coefficient for the minority spin electrons was 0.7 that found for the majority spin electrons. The total transmitted current was of the order of $10^{-5}$ of the primary beam. By cesiating both sides of the film, this transmission ratio was increased to $3 \times 10^{-4}$ and a Sherman function of $\mathrm{S}=0.4$ was measured (van der Sluijs et al 1994).

\subsection{Using the spin polarimeter}

Before closing our discussion of the experimental details we offer a brief introduction to the practicalities of a spin-polarization measurement. A more detailed discussion can be found elsewhere (Kessler 1985)

In order to measure a spin polarization it is necessary to define a quantization direction for the spin of the electrons. In studies of ferromagnetic or ferrimagnetic systems this is achieved by ensuring the sample is magnetically saturated. As an example, ferromagnetic thin films can be magnetized by discharging a current pulse through a small coil in close proximity to the film. As we discuss later, it is also possible to perform spin polarization measurements on antiferromagnetic or even non-magnetic systems when spin-orbit effects are present. In this case the quantization direction is 
defined by the polarization vector of the incident light with the latter being circularly polarized.

To avoid any error being introduced into the measurement by apparatus asymmetries it is necessary, in the case of ferromagnetic systems, to make two measurements of the spin polarization, one with the sample magnetized 'up' $\left(I_{L}^{+}, I_{R}^{+}\right)$and one with the sample magnetized 'down' $\left(I_{L}^{-}, I_{R}^{-}\right)$. Here $I_{\mathrm{L}}$ and $I_{\mathrm{R}}$ represent the number of electrons scattered from the target into the left and right channels, respectively. Assuming that the incident beam does not move between the '+' and '-' measurements, any instrumental asymmetry derived from a misalignment of the beam incident on the scattering target can then be removed by combining the four measurements such that the true spin polarization $P$ is given by

$$
P=\frac{1}{S} \frac{\sqrt{I_{L}^{+} I_{R}^{-}}-\sqrt{I_{L}^{-} I_{R}^{+}}}{\sqrt{I_{L}^{+} I_{R}^{-}}+\sqrt{I_{L}^{-} I_{R}^{+}}}
$$

where as before $S$ represents the Sherman function of the instrument. The measured intensities combined in this manner remove, to first order, any instrumental asymmetry. The two measurements, ' + ' and '-', may have different count rates without affecting the measured polarization. Assuming that there is not an asymmetry that changes with the two magnetization directions, the instrumental asymmetry $A$ is given by

$$
A=\frac{\sqrt{I_{L}^{+} I_{L}^{-}}}{\sqrt{I_{R}^{+} I_{R}^{-}}}
$$

The individual spin-up and spin-down spectra are obtained from the polarization $P$ by

$$
I^{\uparrow}=\langle I\rangle(1+P) \quad I^{\downarrow}=\langle I\rangle(1-P)
$$

where 


$$
\langle I\rangle=\frac{I_{L}^{+}+I_{L}^{-}+I_{R}^{+}+I_{R}^{-}}{4}
$$

In studies employing incident circularly polarized light equations 13-16 still apply but $(+,-)$ represent the direction of polarization of the incident light.

\section{Some recent applications of SPPES}

In the following we highlight some recent applications of spin-polarized photoemission to problems in condensed matter physics. In particular we highlight some of the latest developments in the study of spin-polarized surface states and quantum well states, we examine the application of high resolution spin polarized photoelectron spectroscopy (SPPES) to the study of spin dependent self-energy effects and finally we

review some recent studies of magnetic oxide systems and ferromagnetic/oxide interfaces. The latter oxide systems present a range of exotic phenomena and are currently the subject of intense research activity with respect to spin functionality in spintronics. Primarily we will restrict our discussion to studies of materials that display magnetic properties. However we will also review the application of SPPES to the study of the properties of the cuprates, the basis of the high Tc superconductors.

\subsection{Spin-polarized surface states.}

Discussed in detail elsewhere (Johnson, 1997), there have previously been a number of studies of spin-polarized surface states. In general the spin polarization of the states investigated in those studies reflected the reduced dimensionality present at the surface or solid/vacuum interface of ferromagnetic materials. Examples include states 
localized on the $\mathrm{Fe}(001)$ (Brookes et al., 1990) and Fe(110) surfaces (Vescovo et al., 1993).

It has recently been shown that electronic states at the surface of certain nonmagnetic materials can also display a measurable spin polarization. In this case the spinpolarization reflects a spin-orbit interaction. Shown in figure 6, a state showing this property was first identified on the surface of $\mathrm{Au}(111)$.(LaShell et al., 1996) In a high resolution photoemission study these authors found that an s-p derived Shockley state displaying parabolic dispersion actually split into two bands as the state moved away from the center of the zone. The authors concluded that the splitting reflected the spinorbit term in the Hamiltonian given by

$$
H_{S O}=\frac{\hbar}{4 m^{2} c^{2}} \underline{\sigma} \cdot(\underline{\nabla} V x \underline{p})
$$

where $\underline{\sigma}$ is the Pauli spin operator and, $\underline{\nabla}$ is the local potential gradient and $\underline{p}$ is the electron momentum. The lack of inversion symmetry at the surface allows this spin-orbit interaction to lift the degeneracy between the two spin states. Similar results and hence confirmation were found in a later study of $\mathrm{Au}(111)$ but not $\operatorname{Ag}(111)$. (Nicolay et al., 2001)

Examination of equation (17) shows that the energy of an electron depends on its spin direction and its momentum. With a given momentum the effect with reverses for different spin directions and for a given spin the effect reverses either side of the zone center. Such effects were confirmed in spin resolved studies of a surface state on the W(110) surface (Hochstrasser et al, 2002) and for the $\mathrm{Au}(111)$ surface state (Hoesch et al., 2004). The results of the latter study are shown in figure 7 where the sign convention (spin-up vs spin-down) is defined in a spin coordinate axis that is the counter-clockwise 
tangent to the Fermi surface contour. The effect has also been reported in a study of the magnetic Gadolinium (0001) surface state that we discuss in detail below (Krupin et al., 2005) In their study Krupin et al. reported the observation of the "Rashba effect" and reported that it was enhanced upon oxidation of the surface.

As Hoesch et al. note, such an effect will be unobservable in the bulk of a material such as gold due to the combined restrictions of the time reversal-symmetry $E^{\uparrow}(\underline{k})=E^{\downarrow}(-\underline{k})$ and the inversion symmetry $E^{\uparrow}(\underline{k})=E^{\uparrow}(-\underline{k})$ leading to the Kramers degeneracy $E^{\uparrow}(\underline{k})=E^{\downarrow}(\underline{k})$.

\subsection{Thin Films and Magnetic Multilayers}

Magnetic multilayers and related thin films have been an important area of study in magnetism. This interest reflects the many potential technological applications of the giant magneto resistance (GMR) property observed in the multilayers. Indeed, this effect first discovered in 1987 by Fert and Grünberg, has already seen widespread use in a number of commercial applications.

GMR is observed in metallic multilayes consisting of ferromagnetic layers separated by non-magnetic spacer layers Depending on the thickness of the spacer layer the adjacent ferromagnetic layers are aligned either ferromagnetically or antiferromagnetically. In the latter configuration a magnetic field may be applied to realign the magnetic layers and thereby modify the spin-dependent transport or resistance

through the system. It is this ability to substantially modify the spin-dependent resistance in these "GMR" materials that makes them so important. 
In order to understand this phenomenon we must first understand the factors that determine the different alignments of the adjacent magnetic layers. In fact the spacer layer thickness that results in the antiferromagnetic alignment can correspond to several atomic layers. Insight into this property comes from the recognition that the system can be treated as a series of 1-dimensinal quantum wells. Within these wells the electronic structure will be quantized with an energy separation that reflects the width or thickness of the spacer layer. Let us consider this quantization. In the vicinity of the interface with the adjacent magnetic layer the wavefunction of the electron state within the well takes the form

$$
\Psi=e^{-i k z}+r_{c} e^{i \phi_{c}} e^{i k z}
$$

where $r_{c} e^{i \phi_{c}}$ represents the complex reflectivity at the interface with the substrate and $\phi_{c}$ gives the phase change of the wavefunction. A stationary or bound state exists whenever the total phase change within the well is given by the Bohr-Sommerfeld quantization condition

$$
2 \phi_{c}+2 k m a=2 \pi n
$$

Here we are considering a well bound by two adjacent magnetic layers and hence two identical interfaces. $2 \mathrm{kma}$ represents the phase change accumulated on the round trip across a well consisting of $m$ atomic layers with interlayer spacing $a$. Thus we see that as the number of layers changes, the quantization condition changes and the energy of the stationary state changes to accommodate additional nodes within the wavefunction. Modifying equation (19) accordingly we obtain

$$
\phi_{C}+(k+\Delta k)(m+1) a=\pi(n+1)
$$


As we will see later, the spin dependent scattering at the interface results in quantum well states showing a strong spin polarization. As the thickness of the nonmagnetic layer changes the quantum well states move to lower energy and cross the Fermi level with a well defined periodicity. The total energy of the system is at a maximum when an occupied quantum well state is sitting right at the Fermi level. Because the states are spin polarized the system can achieve a lower total energy by having adjacent ferromagnetic layers realign to an anti-ferromagnetic configuration. By doing so the quantum well state is allowed to "leak" away through one interface and the total energy is reduced.

The above represents a quantum well description of the interlayer exchange coupling. In a more detailed analysis of this picture, Smith et al (1994) concluded that the appropriate quantum numbers for classifying the quantum well states were not $n$, the number of nodes, or $m$, the number of layers, but rather $v=(m-n)$ as illustrated in figure 8 for silver films deposited on an $\mathrm{Fe}(001)$ substrate. In this classification scheme the thickness against energy relationship may be written

$$
\frac{D_{v}(E)}{a}=\frac{\phi_{c}(E) / \pi+v}{\left(1-k(E) / k_{B Z}\right)}
$$

where $\mathrm{k}_{\mathrm{BZ}}=\pi / \mathrm{a}$ represents the zone boundary wavevector and $D=$ ma. A quantum well state crosses the Fermi level each time $v$ increments by one. It is clear from equation (21) that the Fermi surface will therefore be sampled every $\Delta \mathrm{m}$ layers such that

$$
\Delta \mathrm{m}=\left(1-k_{F} / k_{B Z}\right)^{-1}
$$

where $k_{\mathrm{F}}$ represents the Fermi wavevector. Recognizing that $2\left(k_{\mathrm{BZ}}-k_{\mathrm{F}}\right)$ gives the associated bulk Fermi surface spanning vectors, $q$, one immediately retrieves from 
equation (22) a period length $\Delta$ ma given by $2 \pi / \mathrm{q}$ identical to that obtained by Bruno and Chappert (1991) in an analytical description of the thickness dependence of the oscillatory interlayer exchange coupling in magnetic multilayers.

With a relatively short mean free path for the escaping electrons photoemission does not lend itself to direct studies of the electronic structure of a multilayer. As such the spin polarized photoemission studies relevant to these materials are studies that have focused on the properties of the associated overlayers or thin films. Here we refer to, for instance, silver films on $\mathrm{Fe}(001)$ substrates and copper films on $\mathrm{Co}(001)$ substrates.

The first study that identified the role of spin-dependent interfacial scattering was a study of the quantum well states that form in silver films deposited on an $\mathrm{Fe}(001)$ substrate (Brookes et al 1991). In the study the authors identified an interface state or quantum well state characterizing a monolayer thick silver film. With increasing thickness this state evolves into a series of states that move up to and through the Fermi level as shown in figure 9 . The movement to lower binding energy simply reflects the fact that with each new layer the wavefunction describing the quantum well state has to gain an extra half-wavelength to accommodate the new atomic potential (Smith et al 1994). We can use the same quantization condition given above for the multilayers but now the $\phi_{c}$ of one interface is replaced by the $\phi_{B}$ describing the phase shift of the wavefunction at the solid/vacuum interface. Thus the quantization condition of equation (19) now becomes

$$
\phi_{C}+2 k m a+\phi_{B}=2 \pi n
$$

The spin-polarized photoemission study of Brookes et al (1991) revealed that the Ag quantum well states are highly spin-polarized, preferentially with minority spin. As 
discussed earlier the observation of a strong spin polarization can be explained in terms of the spin-dependent reflectivities at the interface with the ferromagnetic substrate. If one considers the spin-dependent bandstructure of iron in the $I \mathrm{H}$ direction, figure 10, one observes that in the minority spin band structure a s-d hybridization gap exists in the vicinity of the Fermi level. At binding energies corresponding to this gap, the propagating waves will be reflected back into the silver layer. Thus, it is the gap that defines the degree of confinement of the minority spin quantum well state. In the majority spin band structure, on the other hand, the hybridization gap is displaced to higher binding energies and the majority spin states in the silver layer in the vicinity of the Fermi level will be less strongly confined. The quantum well states that survive in the silver layer will, therefore, preferentially carry minority spin as observed experimentally.

Subsequent inverse photoemission studies of the $\mathrm{Ag} / \mathrm{Fe}(001)$ system tracked the quantum well states as they continued to evolve above the Fermi level eventually converging on the bulk $X_{4}^{\prime}$ critical point (Ortega and Himpsel 1992, Ortega et al 1993). These studies clearly demonstrated in this and a number of other systems that the Fermi surface was sampled by the quantum well states with a frequency identical to that observed for the oscillatory exchange coupling in the associated multilayers. This is illustrated in figure 11 where the intensity observed at the Fermi level in inverse photoemission studies of copper films deposited on a $\mathrm{Co}(001)$ substrate is compared with the oscillatory coupling observed in the associated $\mathrm{Cu} / \mathrm{Co}(001)$ multilayers.

An interesting question arises as to whether or not majority spin quantum well states exist in the spin-polarized photoemission spectra recorded from these films. Examination of figure 10 shows that in the Fe majority spin band structure the top of the 
s-d hybridization gap falls only $0.8 \mathrm{eV}$ below the Fermi level and the top of the Ag dbands falls $3.0 \mathrm{eV}$ below. Thus one might anticipate seeing strong majority spin states in the region above the silver d-bands up to $0.8 \mathrm{eV}$ below the Fermi level. In fact such states are predicted in calculations using both the phase model approach of equation (23) and the tight-binding method (Smith et al 1994) and ab initio calculations of the layered KKR type (Crampin et al 1996).

The spin-polarized photoemission study of $\mathrm{Ag}$ on $\mathrm{Fe}(001)$ (Brookes et al 1991) represented a study of the spin-polarization effects in very thin films. Indeed, at the monolayer limit it is probably more reasonable to describe the induced state as an interface state as we have discussed earlier in section 6.2. Two studies have examined the spin polarization characteristics of quantum well states in thicker films. In both cases the studies were of copper films deposited on a fcc $\mathrm{Co}(001)$ substrate (Garrison et al 1993, Carbone et al 1993). As in the earlier Ag/Fe (001) study, the quantum well states shown in figure 12 were found to be highly spin polarized with minority spin. However, now the $\mathrm{Cu}$ films were grown to thicknesses of the order of six atomic layers or more. In fact in a more recent study Carbone et al (1996) reported the observation of quantum well states in copper films of the order of 50 atomic layers thick. At such thicknesses the escape depth of the photoelectrons ensures that the spin-polarization information in the spectrum clearly reflects emission from the $\mathrm{Cu}$ film itself rather than the cobalt substrate. In their study, Carbone et al also claimed the observation of majority spin quantum well states with considerably less intensity than the minority spin counterparts. They determined an exchange splitting of $0.15 \mathrm{eV}$ between the two spin components. 
In order to obtain a better understanding of the photoemission spectra in their study Garrison et al (1993) calculated the electronic structure of the different thickness copper films using a spin-dependent tight-binding scheme in a slab formulation. These tight binding calculations were carried out using an effective Hamiltonian of the form

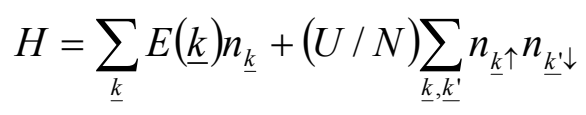

Where the first term reflects the non-magnetic band structure and the second term represents the modification due to the on site spin-dependent potential $U$. The latter was simply taken as the effective Stoner parameter. Using such an approach the authors sought a self-consistent solution such that for each layer $\Delta_{l}=U_{l} m_{l}$ where $\Delta_{l}$ is the layerdependent splitting introduced into the d orbitals, $U_{l}$ the layer dependent Stoner parameter and $m_{l}$ the calculated moment for each layer. Based on their calculation Garrison et al. concluded that the quantum well states have significant $\mathrm{d}$ as well as $\mathrm{sp}$ character. This interesting observation stems from the fact that, within the $\mathrm{Cu}$ film, as in bulk $\mathrm{Cu}$, the s,p bands will hybridize with the d-bands of the same symmetry. This hybridization results in a small fraction of the d-electrons being carried up to and through the Fermi level and indeed in bulk $\mathrm{Cu}$, approximately $3 \%$ of the d-electron manifold is unoccupied. A similar observation has also been made in a recent ab initio layered KKR calculation of copper films deposited on Co(001) (Van Gelderen et al 1996).

The observation that there is a small spin polarization in the $\mathrm{Cu}$ d-band, and further that this band crosses the Fermi level, is a clear indication that a small magnetic moment of d-character must exist on the $\mathrm{Cu}$ site, an observation that was confirmed in a MCD study of $\mathrm{Cu} / \mathrm{Co}$ multilayers (Samant et al 1994, Held et al 1996). Here the authors, exploiting the dipole selection rules, studied absorption at the $\mathrm{Cu}$ L-edge to obtain site 
and spin specific information on the unoccupied d-bands. The study found a small moment of d-character on the copper sites and further concluded that the largest moments exist in the interface where the hybridization with the neighboring Co layers will be strongest. This observation of a localized interfacial magnetic interaction has also been confirmed in numerous other SPPES studies of the d-bands in overlayer films deposited on ferromagnetic substrates. As a few examples, we cite the $\operatorname{Pd}(111) / \mathrm{Fe}(110)$ interface (Weber et al., 1991, Weber et al., 1992), the Pt/Co(0001) interface (Weber et al., 1993), the $\mathrm{Ag} / \mathrm{Fe}(001)$ interface (Brookes et al., 1994) and $\mathrm{Ru}$ and $\mathrm{Rh}$ deposited on $\mathrm{Co}(0001)$ (Rampe et al., 1995). In general these studies all identified a moment on the interfacial overlayer atoms but the induced polarization rapidly decayed away with deposition of subsequent overlayers.

More recently Qiu and coworkers have continued to investigate the properties of quantum well systems and their relationship to the oscillatory exchange coupling. (Zhang et al., 2000, Qiu and Smith, 2002, Wu et al., 2006). In particular these authors have focused on the thickness dependence of the quantum well electronic structure (Rotenberg et al., 2006) and on the properties of double quantum well structures (Ling et al., 2002, Wu et al., 2006).

There is also the possibility of quantizing the electronic structure of d-bands in a thin film. The role of this has been explored in a SPPES study of chromium films grown on an $\mathrm{Fe}(001)$ whisker with particular reference to the oscillatory exchange coupling in Fe/Cr multilayers (Li et al., 1997). In another PES study, Krajl et al. examined the quantization of the d-bands in silver films grown on an $\mathrm{V}(001)$ substrate (Krajl et al., 2003). The authors were successfully able to explain the quantum well structure both in 
terms of the phase models described above and in terms of tight-binding calculations. It is interesting to speculate on the possible role of quantized d-bands in the multilayer structures. Indeed it is known that scattering from a d-resonance at energy $E_{d}$ can induce a phase shift $\delta_{l}$ such that (Harrison, 1980)

$$
\tan \delta_{l}=\frac{\Gamma}{2\left(E_{d}-E\right)}
$$

Thus the scattering induces a phase shift at energy $E$ that increases from 0 to $\pi$ over the range $\Gamma$ of the resonance. A phase shift of $\pi$ will shift the quantum well states from one thickness dependent branch to the next.

\subsection{Collective excitations and self energy effects}

In recent years the energy and momentum resolution of angle-resolved photoemission have improved to a degree that allows detailed studies of self energy effects. Thus it has become routinely possible to study the lifetime and dispersion modifications associated with a variety of collective modes including phonons, spin excitations, and charge density waves. In magnetic systems, with the added complexity of spin detection, the studies of magnetic systems have been considerably more limited Here we review the only detailed study of spin dependent self energy effects, namely a recent study of gadolinium. The spin dependent electronic structure of this material had previously been studied extensively with spin-resolved photoemission.

The ground state of gadolinium is ferromagnetic with a Curie temperature $T_{C}$ of 293K. The (0001) surface of this material has been shown both theoretically (Wu et al., 1991) and experimentally (Li et al., 1991) to support a surface state derived from the Gd $5 \mathrm{~d}$ orbitals. The state, which is spin polarized through an exchange interaction with the 
localized 4f orbitals has an important history and indeed it was spin-resolved photoemission studies of the surface state that finally confirmed that the surface moments were ferromagnetically aligned with the bulk of the material. (Mulhollan et al., 1992)

The results of a more recent photoemission study of this same state held at $20 \mathrm{~K}$ with both high energy and spin resolution are illustrated in figure 13. (Fedorov et al., 2002) As noted earlier, both experiment (Mulhollan et al., 1992, Li et al., 1993) and theoretical calculations (Wu et al., 1991) indicate that the surface state should be $100 \%$ majority spin, reflecting parallel alignment of the surface and bulk moments. The coexistence of both spin components at the same energy in figure 13 is therefore an intrinsic property of the surface state arising from a combination of spin-orbit and spin exchange processes. A simple model yields a polarization $P=\Delta / \sqrt{\Delta^{2}+\zeta^{2}}$ for each quasiparticle state. With a spin-orbit parameter $\xi=0.3 \mathrm{eV}$ and an exchange splitting $\Delta=$ $0.7 \mathrm{eV}$ at $0 \mathrm{~K}$, we get a spin-orbit induced mixing $\mathrm{R}=\left(\mathrm{n} / \mathrm{n}^{-}\right)=(1-\mathrm{P}) /(1+\mathrm{P}) \sim 5 \%$. This increases to $8 \%$ at $T=150 \mathrm{~K}$ as the exchange splitting between the occupied and unoccupied surface states gets smaller. Fedorov et al., 1994, Weshke et al., 1996)

Fitting the spectra in figure 13 with Lorentzian line shapes shows that the minority spin peak has a larger width than its majority spin counterpart, $116 \mathrm{meV}$ as opposed to $86 \mathrm{meV}$. Removing the contribution from the experimental resolution, these widths become approximately $105 \mathrm{meV}$ in the minority spin channel and $70 \mathrm{meV}$ in the majority channel. Possible decay modes for either spin photohole can involve electronphonon, electron-magnon and electron-electron scattering. Each of these different mechanisms will give distinct spin dependent contributions to the scattering rate. Electron-electron scattering by exchange processes favors the two holes in the final state 
being of opposite spin. (Sinkovic et al., 1995) From consideration of the total density of states in the spin channels, the authors of the spin resolved study estimated the scattering rate from the process to be equal for a majority spin hole and a minority spin hole. The electron-phonon and impurity scattering rate are proportional to the density of states at the hole binding energy for the same spin while the electron-magnon rate is proportional to the density of states for the opposite spin. Since the majority-spin density of states is large while the minority-spin part is small and from consideration of the required momentum transfer, impurity and electron-phonon scattering should be more important in the majority spin channel. The observation that the minority spin channel is broader suggests electron-magnon scattering is the dominant decay mechanism. At $T=0 \mathrm{~K}$, the minority-spin component of a photo-hole can scatter to the majority spin component of a hole state higher in the surface band by emitting a spin wave (tilting the spins of the localized f-electrons). The corresponding spin- flip process is not available to the majority-spin component of the photo-hole at $T=0$ because the localized f-spins have saturated magnetization and are not able to tilt upwards when the hole tilts down. At higher temperatures, inelastic scattering can occur back and forth between the two spin channels mediated by the emission or absorption of magnons, but the minority-spin component always has the higher density of final states to scatter into. An approximate treatment (Allen, 2001) using the " $s-f$ " Hamiltonian (Zener, 1951) found the result

$$
\hbar / \tau(\downarrow)=\frac{\sqrt{3}}{4} \frac{P(\uparrow) m^{*}}{S}\left(\frac{2 J S a}{\hbar}\right)^{2}
$$

for the decay of the minority $(\downarrow)$ spin component due to spin flip scattering with magnon emission. Here $J$ is the $s-f$ exchange parameter giving the exchange splitting $2 \mathrm{JS}=0.65$ measured for the surface state, $\mathrm{m}^{*}=1.21$ is the effective mass measured for the surface 
band, and $\mathrm{P}(\uparrow)=0.87$ is the experimentally measured majority component of the band. With $\mathrm{S}=7 / 2$ and $\mathrm{a}=3.6 \AA, \hbar / \tau(\downarrow)=0.095 \mathrm{eV}$. Conversely, replacement of $\mathrm{P}(\uparrow)$ by $\mathrm{P}(\downarrow)$ $=1-\mathrm{P}(\uparrow)$ gives $\hbar / \tau(\uparrow) \approx 0.014 \mathrm{eV}$ for the majority spin component. Thus at low $T$, the majority spin channel is dominated by electron-phonon scattering whereas the minority spin channel is dominated by electron-magnon scattering. Based on the relative spindependent densities of states it is possible to provide estimates of the contribution of phonon scattering in the two spin channels. These would be $46 \mathrm{meV}$ in the majority spin channel and $10 \mathrm{meV}$ in the minority spin channel, leaving approximately $10 \mathrm{meV}$ in each channel due to impurity scattering. This system has also been studied by the group of Kaindl using scanning tunneling spectroscopy. (Rehbein et al., 2003) They arrived at slightly different values for the different contributions but also concluded that the

occupied majority spin surface state preferentially decays via electron-phonon scattering. The important experimental observation in the study described above is that the occupied minority spin component of that state has a shorter lifetime. The only way that that can occur is through the participation of magnon scattering.

\subsection{Half-metallic ferromagnetic oxides}

Half-metallic ferromagnets (HMF) show in the ideal case a $100 \%$ spin polarization at the Fermi level $E_{F}$. The high polarization reflects a metallic density of states for one spin direction and a band gap for the other spin direction. This class of materials was first discovered via ab-initio calculations by de Groot et al. (de Groot et al., 1983) for Mn-based Heusler alloys, such as NiMnSb. Besides the expectation for exceptional magnetooptical properties, these materials have recently attracted 
considerable attention because of their potential application in the field of spintronics (Pickett and Moodera, 2001). In principle, half-metallic ferromagnets are ideal spin injectors and detectors because under moderate voltage they can carry current of only one spin direction. A wealth of theoretical work has been devoted to the spin-dependent electronic structure of metal-based HMF (Kübler, 2003), (Wurmehl et al., 2005) or zincblende chalcogenide HMF (Mavropoulos et al., 2005). Here we focus on transition metal oxide HMF, the existence of which, again, had all been predicted by ab initio electronic structure calculations. Examples include $\mathrm{Fe}_{3} \mathrm{O}_{4}$ (Yanase and Siratori, 1984, Zhang and Satpathy, 1991), $\mathrm{CrO}_{2}$ (Schwarz, 1986, Korotin et al., 1998), $\mathrm{La}_{1-\mathrm{x}} \mathrm{Sr}_{\mathrm{X}} \mathrm{MnO}_{3}$ (Pickett and Singh, 1996) and $\mathrm{Sr}_{2} \mathrm{FeMoO}_{6}$ (Kobayashi et al., 1998). The experimental challenge ever since has been to prove the half-metallic nature of these ferromagnetic or ferrimagnetic materials. While this task would best be carried out using polycrystalline materials to yield an average over all k-states of the Brillouin zone, the experimental studies have mostly been aimed at using single crystalline material or thin epitaxial films. This was neccessary because the particular experimental techniques used for measuring spin polarization all show a subtle surface sensitivity. Among these are spin-polarized tunnelling into superconductors, superconducting point-contact spectroscopy using Andreev reflection, magnetic tunnel junctions and spin-polarized photoemission. While most of the techniques depend critically on interface qualities, photoemission relies particularly on ultra-clean surfaces. Another crucial point concerns the different nature of the electronic states probed by transport and by photoemission measurements. In this section we give an account of the progress made in the field of oxidic HMF using SPPES. 
i) $\mathrm{Fe}_{3} \mathrm{O}_{4}$ (111), (100): SPPES studies of magnetite $\left(\mathrm{Fe}_{3} \mathrm{O}_{4}\right)$ began in 1975 (Alvarado et al., 1975). The interpretation of the valence-band photoemission spectra has been a subject of controversial debate ever since (Alvarado et al., 1976, Alvarado and Bagus, 1978, Cai et al., 1998, Dedkov et al., 2002a, Dedkov et al., 2004, Fonin et al., 2005). Alvarado et al. performed the first SPPES measurements on cleaved $\mathrm{Fe}_{3} \mathrm{O}_{4}$ (100) single crystals. (Alvarado et al., 1975, 1976, 1978) The spin polarization of the photoelectrons showing a maximum value of $-60 \%$ near the Fermi energy $E_{F}$ was measured at $10 \mathrm{~K}$ using an incident photon energy of $5 \mathrm{eV}$ (Alvarado et al., 1976). From a single-ion-in-a-crystalfield (SICF) model, a maximum value of $P=-66.6 \%$ was obtained for the spin polarization at $T=0 \mathrm{~K}$ (Alvarado et al., 1976, 1978). This was considered to be in agreement with the measured value of $-60 \%$. However, subsequent spin polarization measurements on epitaxial thin films of $\mathrm{Fe}_{3} \mathrm{O}_{4}(111)$ and $\mathrm{Fe}_{3} \mathrm{O}_{4}(100)$ yielded at room temperature, values of $-(80 \pm 5) \%$ and $-(40-55) \%$ near $E_{F}$ respectively (Dedkov et al., 2002a, Morton et al., 2002, Huang et al., 2002, Fonin et al., 2005). Figure 14 shows in the left panel the spin-resolved photoemission intensities ( $\mathbf{\Delta}$ spin up, $\boldsymbol{\nabla}$ spin down) together with the total intensity (•) of $\mathrm{Fe}(110)$ (bottom) and $\mathrm{Fe}_{3} \mathrm{O}_{4}(111)$ (top). The spin polarization is shown in the right panel for $\mathrm{Fe}(110)(\boldsymbol{\bullet})$ and $\mathrm{Fe}_{3} \mathrm{O}_{4}(111)(\bullet)$. In both cases the spin polarization at $E_{F}$ is accidentally $-80 \%$. For $\mathrm{Fe}_{3} \mathrm{O}_{4}(111)$ this provided evidence closer to the theoretically predicted half-metallic nature of magnetite (Yanase and Siratori, 1984, Zhang and Satpathy, 1991), at least for the [111] direction.

For the interface between a $25-\mathrm{nm}$ thick $\mathrm{Fe}_{3} \mathrm{O}_{4}(111)$ thin film and a 2-nm thick $\gamma-\mathrm{Al}_{2} \mathrm{O}_{3}(111)$ layer a negative spin polarization of about $-40 \%$ was found by SPPES (Bataille et al., 2006). 
Interestingly, for the (100)-oriented epitaxial thin films of $\mathrm{Fe}_{3} \mathrm{O}_{4}$ spin polarization values of only $-(40-55) \%$ near $E_{F}$ were obtained (Morton et al., 2002, Huang et al., 2002). These values are significantly lower than the $-100 \%$ predicted by local spin density approximation (LSDA) calculations for the bulk material (Zhang and Satpathy, $1991)$ as well as the $-(80 \pm 5) \%$ measured at room temperature on the (111)-oriented $\mathrm{Fe}_{3} \mathrm{O}_{4}$ surface near $E_{F}$ (Dedkov et al., 2002a). The low value for the (100) surface was ascribed either to surface imperfection (Morton et al., 2002) or to strong electron correlation effects (Huang et al., 2002). Recent measurements of SPPES on epitaxial thin films of $\mathrm{Fe}_{3} \mathrm{O}_{4}(100)$ gave a spin polarization of $-(55 \pm 10) \%$ at $E_{F}$ (Fonin et al., 2005). In an effort to explain this result on the basis of density functional theory (DFT) and $a b$ initio thermodynamics calculations it was discovered that the surface electronic structure of $\mathrm{Fe}_{3} \mathrm{O}_{4}(100)$ differs distinctly from the bulk one. $\mathrm{A}(\sqrt{2} \mathrm{x} \sqrt{2}) R 45^{0}$ wavelike surface atom reconstruction due to a Jahn-Teller effect was found to lead to surface states in the majority spin band gap, resulting in a calculated spin polarization of $-40 \%$ at $E_{F}$ (Fonin et al., 2005). However, for all of these comparisons between experiment and theory it has to be remembered that, except for the latter reference, the electronic structure calculations have been performed for the bulk material without taking account of subtle surface reconstruction and relaxation or charge redistribution effects, particularly for (100) polar surfaces. These surface effects certainly play an important role in VUV-PES and SPPES measurements.

For correlated electron systems, such as $\mathrm{Fe}_{3} \mathrm{O}_{4}$, an atomic configuration-based approach had initially been chosen for the interpretation of the SP-PES data (Alvarado et al., 1976, 1978). Recent investigations (Cai et al., 1998, Dedkov et al., 2002a), however, 
showed that band dispersions (Yanase and Siratori, 1984, Zhang and Satpathy 1991) must be taken into account for the interpretation of photoelectron spectra of $\mathrm{Fe}_{3} \mathrm{O}_{4}$. In particular, surface symmetry-related band dispersions were identified for $\mathrm{Fe}_{3} \mathrm{O}_{4}(111)$. Angle-resolved PES determinations of the electronic band structure of well-ordered $\mathrm{Fe}_{3} \mathrm{O}_{4}(111)$ surfaces using synchrotron radiation (Dedkov et al., 2004) gave direct evidence for surface-Brillouin-zone (SBZ) symmetry-related contributions of the oxygen and iron sublattices. In the $\bar{\Gamma}-\overline{\mathrm{M}}$ direction of the $\mathrm{Fe}_{3} \mathrm{O}_{4}(111) \mathrm{SBZ}$, two types of dispersing states were identified. They originate from a periodic multilayered structure of iron and oxygen ions, with $\mathrm{Fe}^{2+}$ and $\mathrm{Fe}^{3+}$ cations incorporated into the close-packed fcc oxygen sublattice. For $\mathrm{Fe}_{3} \mathrm{O}_{4}(100)$ a "wave-like" atom pattern observed by STM along the [110] direction (Tarrach et al., 1993, Stanka et al., 2000, Fonin et al., 2005) gave the first hint of surface reconstruction effects, which were finally corroborated by DFT calculations (Fonin et al., 2005). Over a broad range of oxygen pressures, the modified B-layer bulk termination, consisting of oxygen and iron $\left(\mathrm{Fe}_{B}\right)$ in octahedral B-sites and showing a pairwise "wave-like" shift of iron atoms perpendicular to the $B$-rows, was identified as lowest energy configuration. As a consequence of this reconstruction, surface states appear in the band gap of the majority spin subband leading to a reduction of the spin polarization to about $-40 \%$ at $E_{F}$ and a loss of half-metallicity of the $\mathrm{Fe}_{3} \mathrm{O}_{4}(100)$ surface. These surface states are a hybridization of $d_{x}{ }^{2}-y^{2}$ states of octahedral iron in the surface layer and $p_{x}, p_{y}$ states of the surface oxygen without a subsurface tetrahedral iron neighbor.

ii) $\mathrm{CrO}_{2}$ (100): In spite of applications in magnetic tapes and potential use for magnetooptical storage, the electronic structure of $\mathrm{CrO}_{2}$ was theoretically predicted only in 1986 
using the local spin density approximation (LSDA) to DFT (Schwarz, 1986). The first SPPES investigations revealed a spin polarization of up to $90 \%$ near $2 \mathrm{eV}$ binding energy below $E_{F}$ (Kämper et al., 1987). No spin analysis using a 100-kV Mott spin detector (Kisker et al., 1982) was feasible at the time between $E_{F}$ and $2 \mathrm{eV}$ binding energy because of the low photoemission intensity. The low emission intensity was attributed to oxygen deficiencies. However, high quality epitaxial thin films of $\mathrm{CrO}_{2}(100)$ grown on $\mathrm{Ti}(100)$ single crystal substrates showed a spin polarization of up to $95 \%$ at $E_{F}$ (Dedkov et al., 2002b). Figure 15 shows in the right panel the spin polarization as a function of binding energy of an epitaxial $\mathrm{CrO}_{2}(100)$ film (a) after $\mathrm{Ar}^{+}$sputtering for 210 secs at 500 $\mathrm{eV}$ and (b) after 750 secs sputtering and an additional annealing treatment at $150{ }^{\circ} \mathrm{C}$ for 12 hrs. The left panel shows spin-polarized photoemission spectra ( $\boldsymbol{\nabla}$ spin down, $\boldsymbol{\Delta}$ spin up) and total photoemission intensity $(\bullet)$. The value of $\mathrm{P}=95 \%$ at $E_{F}$ in figure $15 \mathrm{a}$ agrees with measurements using superconducting point contact spectroscopy (Soulen et al., 1998, Ji et al., 2001). The good agreement between spin polarization values from photoemission and transport measurements has to be attributed to the unsusually strong $2 p(O)-3 d(C r)$ hybridization found in electronic structure calculations based on LSDA+U (Korotin et al., 1998). A value of $90 \%$ spin polarization for unoccupied states above $E_{F}$ was also found using spin-resolved O 1s X-ray absorption spectroscopy (Huang et al., 2003). These data give evidence that the presence of holes in the oxygen band prevents $\mathrm{CrO}_{2}$ from being an insulator and supporting the notion that the material is a self-doped or p-type metal (Korotin et al., 1998).

The shoulder in the photoemission intensity in figure 15a,b, which becomes most pronounced after sputtering for $750 \mathrm{sec}$, has been associated with the localized $\mathrm{Cr}(3 \mathrm{~d})$ 
states. These, however, were found in LSDA+U calculations (Korotin et al., 1998) to be near $1 \mathrm{eV}$ binding energy. The photoemission intensity near $2 \mathrm{eV}$ was more recently attributed to emission from the $\mathrm{Cr}_{2} \mathrm{O}_{3}$ surface layer (Chang et al., 2005). This conclusion was reached on the basis of magnetic circular dichroism (MCD) in resonant photoemission studies with $\mathrm{h} v$ tuned to the $\mathrm{Cr} 2 \mathrm{p}$ absorption edge. The energy positions of the $\mathrm{Cr} 3 \mathrm{~d}$ bands in $\mathrm{CrO}_{2}$ are then determined with no contribution from antiferromagnetic $\mathrm{Cr}_{2} \mathrm{O}_{3}$. However, this conclusion contradicts the finding of the peak at $2 \mathrm{eV}$ binding energy after prolonged sputtering $(750 \mathrm{sec})$, removing all $\mathrm{Cr}_{2} \mathrm{O}_{3}$ (Dedkov et al., 2002b). Hence the peak at $2 \mathrm{eV}$ is of intrinsic origin, most likely associated with the lower Hubbard band (LHB). The upper Hubbard band (UHB) may be evident in the atomic-like $\mathrm{Cr} 3 \mathrm{~d}$ state observed in spin-resolved $\mathrm{O}$ 1s X-ray absorption near $0.6 \mathrm{eV}$ above $E_{F}$ (Huang et al., 2003). These observations combined give a Coulomb correlation energy $\mathrm{U}$ in agreement with the value $\mathrm{U}=3 \mathrm{eV}$ used in LSDA+U electronic structure calculations (Korotin et al., 1998). Further, in agreement with these calculations, a more localized $\mathrm{Cr} 3 \mathrm{~d}$ derived spectral weight near $1 \mathrm{eV}$ is found by MCD in resonant photoemission (Chang et al., 2005). In the most recent bulk sensitive PES measurements using hard X-rays (HAX-PES, hv = $7942 \mathrm{eV}$ ) (Suga, 2006) dispersionless $\mathrm{Cr}(3 \mathrm{~d})$ states were found near $1 \mathrm{eV}$ binding energy. Most interesting in the HAX-PES measurements is the rather small spectral weight near $E_{F}$, similar to the previous VUV-PES data by Kämper et al. (Kämper et al., 1987). This is in strong contrast to a theoretical study using LSDA+DMFT (dynamic mean field theory) and applying the iterated perturbation theory (IPT) as "Anderson-impurity solver" to the multi-orbital case (Craco et al., 2003). In this calculation $\mathrm{CrO}_{2}$ is found to exhibit a rather unusually high density of states at $E_{F}$. The 
authors stress the importance of dynamical correlation leading to a collective orbital Kondo effect and the emergence of a correlated Fermi liquid scale. However, the puzzle of the low intensity or missing Fermi edge in the earlier surface-sensitive VUV (hv = $21.2 \mathrm{eV}$ ) photoemission data (Kämper et al., 1987) has recently been attributed to a surface relaxation of $\mathrm{CrO}_{2}(100)$ deduced from LSDA-based first principles calculations (Hong and Che, 2006). The distorted tetrahedral oxygen coordination of each surface $\mathrm{Cr}$ ion gives rise to an inversion of the $t_{2 g}-e_{g}$ splitting of the $\mathrm{Cr} 3 \mathrm{~d}$ orbitals, with $E_{F}$ lying in a gap between the occupied $e_{g}$ and unoccupied $t_{2 g}$ states for a local electronic structure at the surface. For future photoemission intensity calculations of $\mathrm{CrO}_{2}$ it appears indispensable to apply computational schemes merging the local density approximation (LDA) with dynamical mean-field theory (DMFT) and using quantum Monte-Carlo simulations (QMC) to solve the effective Anderson impurity model of DMFT.

iii) Manganites: The manganite class of materials is unusual not only because of the occurrence of the colossal magnetoresistance (CMR), but also because of its dense granular magnetoresistance, and the development of concepts like double exchange and Jahn-Teller polarons. The rich electronic phase diagrams reflect the fine balance of interactions which determine the electronic ground state. The different competing, novel phases, including orbital order and orbiton excitations, arise from an interaction between different microscopic degrees of freedom, including charge, spin, orbitals and lattice. The most straightforward evidence of a minority spin gap and a concomitant $95 \%$ spin polarization near $E_{F}$ was obtained in $\mathrm{La}_{0.7} \mathrm{Sr}_{0.3} \mathrm{MnO}_{3}$ at $40 \mathrm{~K}$ by means of SPPES (Park et al., 1998). This observation was consistent with first principles calculations of $\mathrm{La}_{1-}$ ${ }_{x} \mathrm{Ca}_{\mathrm{x}} \mathrm{MnO}_{3}$ (Pickett and Singh, 1996). Figure 16 shows the temperature dependence of the 
spin polarization of the $\mathrm{Mn} 3 d$ states, $e_{g}$ at $1.0 \mathrm{eV}$ binding energy and $t_{2 g}$ at $2.2 \mathrm{eV}$ binding energy, obtained with $h v=40 \mathrm{eV}$ (Park et al., 1998). The half-metallic nature of $\mathrm{La}_{0.7} \mathrm{Sr}_{0.3} \mathrm{MnO}_{3}$ had a crucial impact on spin-polarized tunneling into Co through a $\mathrm{SrTiO}_{3}$ tunnel barrier (de Teresa et al., 1999).

\subsection{Ferromagnet-oxide interfaces}

Interfaces between electrodes of itinerant ferromagnets and insulating oxide layers play a decisive role in magnetic tunnel junctions (MTJs), giving rise to the tunnel magnetoresistance (TMR). A most prominent and successful example is $\mathrm{Fe}(100) / \mathrm{MgO}(100) / \mathrm{Fe}(100)$, which was theoretically predicted to yield a TMR of the order of about $1000 \%$ (Butler et al., 2001, Mathon and Umersky, 2001). The presently highest TMR values that have currently been achieved experimentally are $220 \%$ at room temperature (300\% at $4 \mathrm{~K})$ for sputtered and textured $\mathrm{Fe}(100) / \mathrm{MgO}(100) / \mathrm{Fe}(100)$ (Parkin et al., 2004) and $180 \%$ at room temperature for epitaxial, MBE grown $\mathrm{Fe}(100) / \mathrm{MgO}(100) / \mathrm{Fe}(100)$ (Yuasa et al., 2004). Other materials combinations yielded $260 \%$ at RT (361\% after annealing; $403 \%$ at $5 \mathrm{~K}$ ) for $\mathrm{CoFeB} / \mathrm{MgO} / \mathrm{CoFeB}$ (Hayakawa et al., 2005, Lee et al., 2006) and $410 \%$ at room temperature $(507 \%$ at $20 \mathrm{~K})$ for bcc $\mathrm{Co}(001) / \mathrm{MgO}(001) / \mathrm{Co}(001)$ (Yuasa et al., 2006).

i) $\mathrm{Fe} / \mathrm{MgO}$ : However there also appears to be a problem with the formation of a submonolayer (ML) of $\mathrm{FeO}$ at the $\mathrm{Fe} / \mathrm{MgO}$ interface (Meyerheim et al., 2001, Meyerheim et al., 2002), which may be responsible for reduced TMR values (Zhang et al., 2003). The formation of $\mathrm{FeO}$ at the interface of $\mathrm{MBE}$ grown $\mathrm{Fe}(110) / \mathrm{MgO}(111)$ could be identified via STM as well as by SPPES (Dedkov et al., 2006). Figure 17 shows the spin-resolved 
photoemission spectra (left panel) as well as the spin polarization (right panel) for clean $\mathrm{Fe}(110)$ and the same with $2 \AA \mathrm{MgO}$ and $5 \AA \mathrm{MgO}$ overlayers (from bottom to top). With increasing overlayer thickness the polarization is attenuated from $-80 \%$ to $-50 \%$ and $20 \%$, respectively. This attenuation of the spin polarization as a function of thickness of the $\mathrm{MgO}$ overlayer is represented in figure 18.

In figure 18 exponential fits to the experimentally observed depolarization of electrons $\left(P_{0}=23.5 \%\right)$ excited by circularly polarized light $(h v=3.05 \mathrm{eV})$ in germanium after traversing an evaporated overlayer of $\mathrm{Ni}$ (dashed line), $\mathrm{Gd}$ (dotted line), and $\mathrm{Ce}$ (dot-dashed line) are shown. All curves show an exponential dependence of the polarization on the thickness of the overlayer possessing valence $d$-electrons in the vicinity of $E_{F}$. The mean free path for spin-flip scattering decreases as a function of the number of unoccupied $d$ states in the valence band from $\mathrm{Ni}$ via $\mathrm{Gd}$ to $\mathrm{Ce}(\mathrm{Ni}$ : two unoccupied $d$ orbitals, $4 s^{2} 3 d^{8}$; Gd: nine unoccupied $d$ orbitals, $6 s^{2} 5 d^{1}$; Ce: ten unoccupied $d$ orbitals, $6 s^{2} 5 d^{0}$ ) (Meier et al., 1984). The reference curves for the $\mathrm{Gd} / \mathrm{Ge}$ and $\mathrm{Ce} / \mathrm{Ge}$ systems are very close to the experimentally observed sharp decrease of spin polarization in the $\mathrm{MgO} / \mathrm{Fe}(110)$ system and can qualitatively be used as additional argument for the presence of a depolarizing $\mathrm{FeO}$ layer at the $\mathrm{MgO} / \mathrm{Fe}(110)$ interface, i.e. spin scattering into the four unoccupied $d$ orbitals of $\mathrm{Fe}^{2+}\left(3 d^{6}\right)$ in $\mathrm{FeO}$. The comparison is only qualitative with respect to the absolute number of hole states as there may be additional spin-scattering at the $\mathrm{FeO} / \mathrm{MgO}$ interface. The presence of such an $\mathrm{FeO}$ interfacial layer and the increase of the $\mathrm{FeO}$ layer thickness at the $\mathrm{MgO} / \mathrm{Fe}$ interface with increasing $\mathrm{MgO}$ layer thickness is supported by AES and STM measurements (Dedkov et al., 2006). For 
these MTJs values of the TMR at RT of $32 \%(54 \%$ at $1.5 \mathrm{~K})$ were obtained (Guerrero et al., 2005, Hauch et al., 2006).

The influence of $\mathrm{MgO}$ overlayers deposited on 16 ML Fe grown on GaAs(100) has been investigated for direct transitions between different symmetry Fe bulk and final states by means of SPPES (Matthes et al., 2004). A spin-dependent attenuation for direct transitions related to Fe bulk initial states has been observed as a function of the $\mathrm{MgO}$ thickness.

ii) $\alpha-\mathrm{Al}_{2} \mathrm{O}_{3} / \mathrm{Fe}(110)$ : The optimization of the oxidation process of thin $\mathrm{Al}$ films deposited on an Fe(110) surface was characterized in a spin-, angle-, as well as energy-resolved photoemission study (SPARPES) of the valence band (Dedkov et al., 2002c). The spinresolved spectra together with the total intensity and the spin polarization respectively, as a function of the binding energy for $\mathrm{W}(110), \mathrm{Fe}(110), 7 \AA \mathrm{Al}$ on $\mathrm{Fe}(110)$ as well as $\alpha$ $\mathrm{Al}_{2} \mathrm{O}_{3} / \mathrm{Fe}(110)$ are presented in figures 19 (a) and 19(b). For the $7 \AA-\mathrm{Al} / \mathrm{Fe}$ system the spin polarization near $E_{F}$ is decreased from $-80 \%$ characteristic of the clean $\mathrm{Fe}(110)$ surface to about $-(35 \pm 5) \%$. Aluminum has no $d$ electrons in the valence band and, therefore, the scattering of spin-polarized electrons from the valence band of Fe can be described by the spin-averaged scattering cross section (Siegmann, 1994). The spin polarization of photoelectrons for adlayer/ferromagnet systems can be calculated (Siegmann, 1994) by $P=P_{0} \exp (-d \cdot \sigma)$, where $P_{0}=-80 \%$ is the polarization of the pure Fe(110) film, $d$ the thickness of the adlayer ( $7 \AA$ for $\mathrm{Al}$ and $13 \AA$ for $1 M L$ of $\alpha-\mathrm{Al}_{2} \mathrm{O}_{3}$ ), and $\sigma$ the total scattering cross section, which in our case is equal to the spin-independent part of $\sigma, \sigma_{0}=$ $1 / 8 \AA^{-1}$ for both materials ( $\mathrm{Al}$ and $\mathrm{Al}_{2} \mathrm{O}_{3}$ ) without $d$-electrons in the valence band 
(Siegmann, 1994). In this case the spin polarization would be attenuated to (-33\%) which is in reasonably good agreement with the value of $-(35 \pm 5) \%$ observed experimentally.

The spin-resolved spectra following oxidation of the 7- $\AA$ thick Al layer on $\mathrm{Fe}(110)$ via subsequent annealing at $250{ }^{\circ} \mathrm{C}$ are presented in figure 19(a) (top curves). The spin polarization of the $\alpha-\mathrm{Al}_{2} \mathrm{O}_{3} / \mathrm{Fe}(110)$ system decreases to $-(15 \pm 5) \%$ near $E_{F}$. The reason for the decrease of the spin polarization can be due to additional attenuation of the spin-polarized photoelectrons by the $\mathrm{Al}_{2} \mathrm{O}_{3}$ layer with an estimated thickness of 11 $\AA$, close to the thickness of $1 M L$ of $\alpha-\mathrm{Al}_{2} \mathrm{O}_{3}$. The estimated spin polarization of $1 M L \alpha-$ $\mathrm{Al}_{2} \mathrm{O}_{3} / \mathrm{Fe}(110)$ is $-17 \%$, which is in the range of the experimental value of $-(15 \pm 5) \%$. The spin polarization of $-15 \%$ in the $\alpha-\mathrm{Al}_{2} \mathrm{O}_{3} / \mathrm{Fe}(110) / \mathrm{W}(110)$ system obtained by SPARPES is in contrast to the positive value determined by spin-polarized tunneling into superconductors (Merservey and Tedrow, 1994). For the photon energy of $h v=21.2 \mathrm{eV}$, the cross section of the photoemission process from the valence band of Fe is larger for $3 d$ electrons than for $4 s$ electrons. Therefore, an attenuation of the negative spin polarization of $\mathrm{Fe}$ without change in sign, due to the coverage with $\alpha-\mathrm{Al}_{2} \mathrm{O}_{3}$ can be described by the spin-independent part of the scattering cross section (Siegmann, 1994). In tunneling experiments $4 s$ electrons from the valence band of $\mathrm{Fe}$ have a higher tunneling probability in comparison with $3 d$ electrons. It is the participation of the $4 \mathrm{~s}$ electrons that explains the positive sign of the spin polarization as calculated (Tsymbal and Pettifor, 1997) and experimentally observed (Meservey and Tedrow, 1994) for tunneling from the ferromagnet into the superconductor.

\subsection{The Antiferromagnetic Cuprates:}


The origin of the high $T_{c}$ superconductivity observed in the cuprates presents one of the greatest challenges in condensed matter physics today. As such it is particularly important to understand the nature of the low-energy excitations in these materials. It is generally accepted that the superconductivity in the cuprates evolves from a parent insulating state by doping carriers into the 2-dimensional $\mathrm{CuO}_{2}$ planes. The ground state of the parent compound is an antiferromagnetic Mott insulator. With doping, the systems move from the antiferromagnetic state to a regime where superconductivity is possible. Cluster calculations indicate that the ground state associated with the $\mathrm{CuO}_{2}$ planes consists of a linear combination of $3 \mathrm{~d}^{9}$ and $3 \mathrm{~d}^{10} \underline{\mathrm{L}}$ states, and the photoemission final state as a combination of $3 \mathrm{~d}^{8}, 3 \mathrm{~d}^{9} \underline{\mathrm{L}}$ and $3 \mathrm{~d}^{10} \underline{\mathrm{L}}^{2}$ states, where $\underline{\mathrm{L}}$ denotes an oxygen ligand hole orbital. Most theories predict that the $3 \mathrm{~d}^{8}$ states close to the Fermi level are of local singlet character rather than the high spin triplet state favored by Hund's first rule.

To investigate this possibility Tjeng and coworkers have used incident circularly polarized light and spin-resolved the electrons emitted in a resonant photoemission process involving the $2 p$ spin-orbit split core level (L.H. Tjeng et al., 1997, L.H. Tjeng et al., 1999, L.H. Tjeng et al., 2001). The authors of these studies point out the critical importance of initially exciting from the spin-orbit split core level. In the absence of such an excitation the measured polarization from the valence bands would be zero for an antiferromagnetic material. The results of their study of optimally doped $\mathrm{Bi}_{2} \mathrm{Sr}_{2} \mathrm{CaCu}_{2} \mathrm{O}_{8+\delta}$ (Brookes et al., 2001) are shown in figure 20, which shows the spinintegrated photoemission spectrum, the same spectrum resolved into triplet and singlet contributions and the measured spin polarization. The latter polarization, defined as $(\uparrow \uparrow-$ $\uparrow \downarrow) /(\uparrow \uparrow+\uparrow \downarrow)$ where $\uparrow \uparrow$ refers to parallel alignment of the photon spin and electron spin, 
$\sigma^{+} \mathrm{e}^{\uparrow}+\sigma^{-} \mathrm{e}^{\downarrow}$, and $\uparrow \downarrow$ refers anti-parallel alignment of the same, $\sigma^{+} \mathrm{e}^{\downarrow}+\sigma^{-} \mathrm{e}^{\uparrow}$, is calculated to be $5 / 6$ or $83.3 \%$ for pure singlet states and $-1 / 3 * 5 / 6$ or $-27.8 \%$ for triplet states. It is clear from the lower panel of figure 20 that the measured polarization provides strong evidence for the presence of the so-called Zhang-Rice singlets (Zhang and Rice, 1988) in the vicinity of the Fermi level.

\subsection{Summary and future outlook}

We have seen that in the past 20 years the development and application of spinpolarized photoemission has been extensive. Because of its sensitivity to the surface region it has offered a number of new insights into the electronic structure of ferromagnetic surfaces and thin films. Studies of the latter have been particularly relevant to understanding the properties of the GMR materials. Further with the increased photon fluxes now available from synchrotron radiation sources it has become possible to develop new methodologies and extend the technique to the study of non-magnetic and antiferromagnetic materials. We may anticipate still further developments in the future particularly in the area of development of more efficient spin polarimeters. Such developments will allow experiments to be carried out with higher energy resolution than has been achieved to date. Amongst other things this will make accessible studies of the fine details associated with magnetic anisotropies in thin films. We may also anticipate further developments in the combination of spin polarized photoemission and microscopy. This will be particularly relevant as detailed studies of nanoscale systems are pursued, particularly with reference to materials investigated for their potential use in spintronics. 


\section{Acknowledgments}

Peter Johnson would like to acknowledge the many contributions of collaborators to his own work in this area including A. Clarke, N. Brookes, B. Sinkovic, K. Garrison, Y. Chang, A.V. Fedorov, S.L. Hulbert, M. Weinert, P.B. Allen and many others. He would particularly like to thank the late Neville Smith for his continued interest and inspiration. Work at Brookhaven National Laboratory is supported by the Department of Energy under Contract No. DE-AC02-98CH10886. Gernot Güntherodt would like to thank his Ph.D. students Yu.S. Dedkov, M. Fonin, J.O. Hauch and C. König as well as his former group assistant and collaborator U. Rüdiger for the fruitful cooperation. The experimental support by P. Turban and the valuable theoretical support and inspiration by R. Pentcheva are gratefully acknowledged. He is also indebted to S. Suga and his team for a most fruitful collaboration. 


\section{References;-}

Allen, P.B., (2001) Electron spin-flip relaxation by one magnon processes: Application to the gadolinium surface band Physical Review B 63, 214410-214414

Alvarado, S. F., Eib, W., Meier, F., Pierce, D.T., Sattler K., Siegmann, H.C., and

Remeika, J.P., (1975) Observation of Spin-Polarized Electron Levels in Ferrites Physical Review Letters 34, 319-322

Alvarado, S. F., Erbudak, M., and Munz, P., (1976) Final-state effects in the $3 d$ photoelectron spectrum of $\mathrm{Fe}_{3} \mathrm{O}_{4}$ and comparison with $\mathrm{Fe}_{x} \mathrm{O}$ Physical Review B 14, 27402745

Alvarado, S. F. and Bagus, P.S., (1978) Fractional spin polarization in the model of photoemission from localized shells Physics Letters 67A, 397-398

Bataille, A. M., Tagliaferri, A., Gota, S., de Nadaï, C., Moussy, J.-B., Guittet, M.-J., Bouzehouane, K., Petroff, F., Gautier-Soyer, M., and Brookes, N. B., (2006) Negative spin polarization of the $\mathrm{Fe}_{3} \mathrm{O}_{4} /{ }_{-} \mathrm{Al}_{2} \mathrm{O}_{3}$ interface measured by spin-resolved photoemission Physical Review B 73, 172201-172204

Brookes, N.B., Clarke, A., Johnson, P.D., and Weinert, M., (1990) Physical Review B 41 2643-2645

Brookes, N.B., Chang, Y. and Johnson, P.D., (1991) Magnetic interface states and finitesize effects Physical Review Letters 67, 354-357

Brookes, N.B., Chang, Y. and Johnson, P.D. (1994) The Ag/Fe(001) Interface Physical Review B 50 15330-15336 
Brookes, N.B., Ghiringhelli, G., Tjernberg, O., Tjeng, L.H., Mizokawa, T., Li, T.W., and Menovsky, A.A., (2001) Detection of Zhang-Rice Singlets using Spin Polarized Photoemission, Physical Review Letters 87, 237003-237006

Bruno, P. and Chappert, C., (1991) Oscillatory coupling between ferromagnetic layers separated by a nonmagnetic metal spacer Physical Review Letters 67, 1602-1605 Burnett, G.C., Monroe, T.J., and Dunning, F.B., (1994) High-efficiency retardingpotential Mott Polarization Analyzer, Review of Scientific Instruments 65 1893-1896 Butler, W.H., Zhang, X.-G., Schulthess, T.C., and MacLaren, J.M., (2001) Spindependent tunneling conductance of $\mathrm{Fe}|\mathrm{MgO}| \mathrm{Fe}$ sandwiches Physical Review B 63, 054416-054427

Cai, Y.Q., Ritter, M., Weiss, W., and Bradshaw, A.M., (1998) Valence-band structure of epitaxially grown $\mathrm{Fe}_{3} \mathrm{O}_{4}(111)$ films Physical Review B 58, 5043-5051

Carbone, C., Vescovo, E., Rader, O., Gudat, W. and Eberhardt, W., (1993) Exchange split quantum well states of a noble metal film on a magnetic substrate Physical Review Letters 71, 2805-2808

Carbone, C., Vescovo, E., Klasges, R., Sarma, D.D. and Eberhardt, W., (1996) Magnetic quantum size effects in $\mathrm{Cu}$ films on $\mathrm{Co}(100)$ Journal of Magnetism and Magnetic Materials 156, 259-260

Chang, C.F, Huang, D.J., Tanaka, A., Guo, G.Y., Chung, S.C., Kao, S.-T., Shyu, S.G., and Chen, C.T.,(2005) Electronic structure of $\mathrm{CrO}_{2}$ studied by magnetic circular dichroism in resonant photoemission Physical Review B 71, 052407-052410 Craco, L, Laad, M.S. and Mүller-Hartmann, E., (2003) Orbital Kondo Effect in $\mathrm{CrO}_{2}$ : A Combined Local-Spin-Density-Approximation Dynamical-Mean-Field-Theory Study 


\section{Physical Review Letters 90, 237203-237206}

Crampin, S., De Rossi, S. and Ciccaci, F., (1996) Integrity of quantum-well resonances in metallic overlayers Physical Review B 53 13817-13823

Dedkov, Yu.S., Rüdiger, U., and Güntherodt, G., (2002a) Evidence for the half-metallic ferromagnetic state of $\mathrm{Fe}_{3} \mathrm{O}_{4}$ by spin-resolved photoelectron spectroscopy Physical Review B 65, 064417-064421

Dedkov, Yu.S., Fonine, M., Konig, C., Rüdiger, U., Güntherodt, G., Senz, S., and Hesse, D., (2002b) Room -temperature observation of high-spin polarization of epitaxial $\mathrm{CrO}_{2}(100)$ island films at the Fermi energy Applied Physics Letters 80, 4181-4183

Dedkov, Yu.S., Fonine, M., Vyalikh, D.V., Hauch, J.O., Molodtsov, S.L., Rüdiger, U., and Güntherodt, G., (2004) Electronic structure of the $\mathrm{Fe}_{3} \mathrm{O}_{4}(111)$ surface Physical Review B 70, 073405-073408

Dedkov, Yu.S., Fonin, M., Rüdiger, U., and Güntherodt, G., (2006) Spin-resolved photoelectron spectroscopy of the $\mathrm{MgO} / \mathrm{Fe}(110)$ system Appl. Phys. A 82, 489-493 Feder R (1985) Polarized Electrons in Surface Physics (Singapore: World Scientific) Fedorov, A.V., Starke, K. and Kaindl, G., (1994) Temperature-dependent exchange splitting of unoccupied electronic states in $\operatorname{Gd}(0001)$ Physical Review B 50, 2739-2742

Fedorov, A.V., Valla, T., Liu, F., Johnson, P.D., Weinert, M. and Allen, P.B., (2002) Spin-resolved photoemission study of photohole lifetimes in ferromagnetic gadolinium, Physical Review B 65, 212409 (2002)

Fonin, M., Pentcheva, R., Dedkov, Yu.S., Sperlich, M., Vyalikh, D.V., Scheffler, M., Rüdiger, U. and Güntherodt, G., (2005) Surface electronic structure of the $\mathrm{Fe}_{3} \mathrm{O}_{4}(100)$ : Evidence of a half-metal to metal transition Physical Review B 72, 104436-104443 
Garrison, K., Chang, Y. and Johnson, P.D., (1993) Spin polarization of quantum well states in copper thin films deposited on a $\mathrm{Co}(001)$ substrate Physical Review Letters 71 $2801-2804$

Graf, J., Jozwiak, C., Schmid, A.K., Hussain, Z., and Lanzara, A., (2005) Mapping the spin-dependent electron reflectivity of Fe and Co ferromagnetic thin films Physical Review B 71 144429-144433

de Groot R.A., Mueller, F.M., van Engen, P.G., and Buschow, K.H.J., (1983) New Class of Materials: Half-Metallic Ferromagnets Physical Review Letters 50, 2024-2027 Guerrero, R., Aliev, F.G., Villar, R., Hauch, J., Fraune, M., Güntherodt, G., Rott, K., Brückl, H., and Reiss, G., (2005) Low-frequency noise and tunneling magnetoresistance in $\mathrm{Fe}(110) / \mathrm{MgO}(111) / \mathrm{Fe}(110)$ epitaxial magnetic tunnel junctions Applied Physics Letters 87, 042501-042503

Harrison, W.A., (1980) Electronic Structure and the Properties of Solids (Freeman, San Francisco)

Hauch, J.O., Fraune, M., Güntherodt, G., Fonin, M., Rüdiger, U., Turban, P., Guerrero, R., Aliev, F.G., and Mayer, J., (2006) Applied Physics Letters, submitted

Hayakawa, J., Ikeda, S., Matsukura, F., Takahashi, H., and Ohno, H., (2005) Dependence of Giant Tunnel Magnetoresistance of Sputtered $\mathrm{CoFeB} / \mathrm{MgO} / \mathrm{CoFeB}$ Magnetic Tunnel Junctions on MgO Barrier Thickness and Annealing Temperature Japanese Journal of Applied Physics 44, L587-L589

Held, G.A., Samant, M.G., Stohr, J., Parkin, S.S.P., Hermsmeier, B.D., van Schilfgarde, M. and Nakajima, R., (1996) X-ray magnetic circular dichroism study of the induced spin 
polarization of $\mathrm{Cu}$ in $\mathrm{Co} / \mathrm{Cu}$ and $\mathrm{Fe} / \mathrm{Cu}$ multilayers Zeitschrift fur Physik-Condensed Matter B 100 335-341

Hochstrasser, M., Tobin, J.G., Rotenberg, Eli and Kevan, S.D., (2002) Spin-Resolved Photoemission of Surface States of W(110)-(1x1)H Physical Review Letters $89216802-$ 216805

Hoesch, M., Muntwiler, M., Petrov, V.N., Hengsberger, M., Patthey, L., Shi, M., Falub, M., Greber, T. and Osterwalder, J., (2004) Spin structure of the Shockley surface state on Au(111) Physical Review B 69, 241401-241404

Hong, F. and Che, J.G., (2006) Low Photoemission Intensity near $E_{\mathrm{F}}$ Induced by the Surface Relaxed Structure of $\mathrm{CrO}_{2}(001)$ Physical Review Letters 96, 167206-167209 Huang, D-J. and Johnson, P.D., Unpublished.

Huang, D.J., Wu, W.P., Chen, J., Chang, C.F., Chung, S.C., Yuri, M., Lin, H.-J, Johnson, P.D. and Chen C.T., (2002) Performance of a Mott detector for undulator-based spinresolved spectroscopy Review of Scientific Instruments 73, 3778-3783

Huang, D.J., Chang, C.F., Chen, J., Tjeng, L.H., Rata, A.D., Wu, W.P., Chung, S.C., Lin, H.J., Hibma, T., and Chen, C.T., (2002) Spin-resolved photoemission studies of epitaxial $\mathrm{Fe}_{3} \mathrm{O}_{4}\left(\begin{array}{lll}1 & 0 & 0\end{array}\right)$ thin films Journal of Magnetism and Magnetic Materials 239, 261-265 Huang, D.J., Tjeng, L. H., Chen, J., Chang, C. F., Wu, W. P., Chung, S.C., Tanaka, A., Hüfner, S., (2003) Photoelectron Spectroscopy (Springer, Berlin)

Guo, G. Y., Lin, H.-J., Shyu, S. G., Wu, C. C., and Chen, C. T., (2003) Anomalous spin polarization and dualistic electronic nature of $\mathrm{CrO}_{2}$ Physical Review B 67, 214419214423

Ji, Y., Strijkers, G. J., Yang, F. Y., Chien, C. L., Byers, J. M., Anguelouch, A., Xiao, G., 
and Gupta, A., (2001) Determination of the Spin Polarization of Half-Metallic $\mathrm{CrO}_{2}$ by Point Contact Andreev Reflection Physical Review Letters 86, 5585-5588

Johnson, P.D., Brookes, N.B., Hulbert, S.L., Klaffky, R., Clarke, A., Sinkovic, B., Smith, N.V., Celotta, R., Kelly, M.H., Pierce, D.T., Scheinfein, M.R., Waclawaki, B.J., and Howells, M.R., (1992) Spin-polarized photoemission spectroscopy of magnetic surfaces using undulator radiation Review of Scientific Instruments 63, 1902-1908 Johnson, P.D., (1997) Spin-Polarized Photoemission, Reports on Progress in Physics 60, $1217-1304$

Kämper, K.-P., Schmitt, W., Güntherodt, G., Gambino, R.J., and Ruf, R., (1987) $\mathrm{CrO}_{2--}$ A New Half-Metallic Ferromagnet? Physical Review Letters 59, 2788-2791

Kessler, K., (1985) Polarized Electrons Springer, Berlin

Kevan S D (1992) Angle-Resolved Photoemission (Amsterdam: Elsevier)

Kirschner, J. and Feder, R., (1979) Spin Polarization in Double Diffraction of LowEnergy Electrons from W(001): Experiment and Theory Physical Review Letters 42, 1008-1011

Kisker, E., Clauberg, R. and Gudat W., (1982) Electron spectrometer for spin-polarized angle- and energy-resolved photoemission from ferromagnets Review of Scientific Instruments 53, 1137-1144

Kisker E (1987) Metallic Magnetism ed H Capellmann (Berlin: Springer) p 57 Kobayashi K.-I., Kimura, T., Sawada, H., Terakura, K., and Tokura, Y., (1998) Roomtemperature magnetoresistance in an oxide material with an ordered double-perovskite structure Nature (London) 395, 677-680

Korotin, M. A., Anisimov, V. I., Khomskii, D. I., and Sawatzky, G. A., (1998) $\mathrm{CrO}_{2}$ : A 
Self-Doped Double Exchange Ferromagnet Physical Review Letters 80, 4305-4308

Kralj, M., Pervan, P., Milun, M., Valla, T., Johnson, P.D., and Woodruff, D.P., (2003)

d-band quantum well states in ultrathin silver films on V(100) Physical Review B 68 $245413-245420$

Krupin, O., Bihlmayer, G., Starke, K., Gorovikov, S., Prieto, J.E., Dobrich, K., Blugel, S. and Kaindl, G., (2005) Rashba effect at magnetic metal surfaces, Physical Review B 71, 201403-201406

Kübler J., (2003) Curie temperatures of zinc-blende half-metallic ferromagnets Physical Review B 67, 220403-220406

LaShell, S., McDougall, B.A. and Jensen, E.A., (1996) Spin Splitting of an Au(111)

Surface State Band Observed with Angle-Resolved Photoelectron Spectroscopy, Physical Review Letters 77, 3419-3422

Lassailly, Y., Drouhin, H-J., van der Sluijs, J., Lampel, G., and Marliere, C., (1994) Spindependent transmission of low-energy electrons through ultrathin magnetic layers Physical Review B 50 13054-13057

Lee, Y.M., Hayakawa, J., Ikeda, S., Matsukura, F., and Ohno, H., (2006) Giant tunnel magnetoresistance and high annealing stability in $\mathrm{CoFeB} / \mathrm{MgO} / \mathrm{CoFeB}$ magnetic tunnel junctions with synthetic pinned layer Applied Physics Letters 89, 042506-042508

Li, D., Hutchings, W., Dowben, P.A., Hwang, C., Wu, R.T., Onellion, M., Andrews, A.B. and Erskine, J.L., (1991) Angle-resolved photoemission evidence for a Gd(0001) surface state Journal of Magnetism and Magnetic Materials 99, 85-90

Li, D., Zhang, J., Dowben, P.A., Garrison, K., Johnson, P.D., Tang, H., Walker, T.G., Hopster, H., Scott, J.C., Weller, D. and Pappas, D.P., (1993) Materials Research Society 


\section{Proceedings 313, 451}

Li, D., Pearson, J., Bader, S.D., Vescovo, E., Huang, D-J., Johnson, P.D. and Heinrich, B. (1997) $k$-Space Origin of the Long-Period Oscillation in Fe/Cr Multilayers: A Photoemission Study of Epitaxial Cr Grown on an Fe(100) Whisker Physical Review Letters 78 1154-1157

Ling, W.L., Rotenberg, Eli, Choi, H.C., Wolfe, J.H., Toyama, F., Paik, S., Smith, N.V., and Qiu, Z.Q., (2002) Double quantum well states in $\mathrm{Cu} / \mathrm{Co} / \mathrm{Cu}$ grown on $\mathrm{Co}(001)$, Physical Review B 65, 113406-115409

Mathon, J. and Umerski, A., (2001) Theory of tunneling magnetoresistance of an epitaxial Fe/MgO/Fe(001) junction Physical Review B 63, 220403-220406

Matthes, F., Tong, L.-N., and Schneider, C.M., (2004) Spin-polarized photoemission spectroscopy of the $\mathrm{MgO} / \mathrm{Fe}$ interface on $\mathrm{GaAs}(100)$ Journal of Applied Physics 95, $7240-7242$

Mavropoulos, Ph., M. Ležaić, and S. Blügel, (2005) Half-metallic ferromagnets for magnetic tunnel junctions by ab initio calculations Physical Review B 72, 174428174433

Meier, F., Bona, G.L., and Hüfner, S., (1984) Experimental Determination of Exchange Constants by Spin-Polarized Photoemission Physical Review Letters 52, 1152-1155 Merservey, R. and Tedrow, P.M., (1994) Spin-polarized electron tunneling Physics Reports 238, 173-243

Meyerheim, H.L., Popescu, R., Kirschner, J., Jedrecy, N., Sauvage-Simkin, M., Heinrich, B., and Pinchaux, R., (2001) Geometrical and Compositional Structure at Metal-Oxide Interfaces: $\mathrm{MgO}$ on $\mathrm{Fe}(001)$ Physical Review Letters 87, 076102-076105 
Meyerheim, H.L., Popescu, R., Jedrecy, N., Vedpathak, M., Sauvage-Simkin, M., Pinchaux, R., Heinrich, B., and Kirschner, J., (2002) Surface x-ray diffraction analysis of the $\mathrm{MgO} / \mathrm{Fe}(001)$ interface: Evidence for an FeO layer Physical Review B 65, 144433144439

Morton, S.A., Waddill, G.D., Kim, S., Schuller, I.K., Chambers, S.A., and Tobin, J.G., (2002) Spin-resolved photoelectron spectroscopy of $\mathrm{Fe}_{3} \mathrm{O}_{4}$ Surface Science 513, L451-

\section{L457}

Mulhollan, G.A., Garrison, K. and Erskine, J.L., (1992) Surface magnetism of Gd(0001): Evidence of ferromagnetic coupling to bulk Physical Review Letters 69, 3240-3243

Nicolay, G., Reinert, F., Hufner, S., and Blaha, P., (2001) Spin-orbit splitting of the Lgap surface state on $\mathrm{Au}(111)$ and $\mathrm{Ag}(111)$ Physical Review B 65, 033407-033410

Ortega, J.E. and Himpsel, F.J., (1992) Quantum well states as mediators of magnetic coupling in superlattices Physical Review Letters 69, 844-847

Ortega, J.E., Himpsel, F.J., Mankey, G.J. and Willis, R.F., (1993) Quantum-well states and magnetic coupling between ferromagnets through a noble-metal layer Physical Review B 47, 1540-1552

Park, J.-H., Vescovo, E., Kim, H.-J., Kwon, C., Ramesh, R., and Venkatesan, T., (1998) Magnetic Properties at Surface Boundary of a Half-Metallic Ferromagnet $\mathrm{La}_{0.7} \mathrm{Sr}_{0.3} \mathrm{MnO}_{3}$ Physical Review Letters 81, 1953-1956; (1998) Direct evidence for a half-metallic Ferromagnet Nature (London) 392, 794-796 Parkin, S.S.P., Kaiser, C., Panchula, A., Rice, P.M., Hughes, B., Samant, M., and Yang, S.-H. (2004) Giant tunnelling magnetoresistance at room temperature with $\mathrm{MgO}$ (100) tunnel barriers Nature Materials 3, 862-867 
Pickett, W. E., and Singh, D. J., (1996) Electronic structure and half-metallic transport in the $\mathrm{La}_{1-x} \mathrm{Ca}_{x} \mathrm{MnO}_{3}$ system Physical Review B 53, 1146-1160

Pickett, W.E. and Moodera, J.S., (2001) Half metallic magnets Physics Today 54, 39-44

Pierce, D.T., Celotta, R.J., Kelley, M.H. and Unguris, J., (1988) Electron spin

polarization analyzers for use with synchrotron radiation Nuclear Instruments and

Methods in Physics Research Section A: Accelerators, Spectrometers, Detectors and Associated Equipment 266, 550-559

Qiu, Z.Q., Pearson, J. and Bader, S.D., (1992) Oscillatory interlayer magnetic coupling of wedged $\mathrm{Co} / \mathrm{Cu} / \mathrm{Co}$ sandwiches grown on $\mathrm{Cu}(100)$ by molecular beam epitaxy, Physical Review B 46 8659-8662

Qiu, Z.Q. and Smith, N.V. (2002) Quantum Well States and Oscillatory Magnetic Interlayer Coupling, Journal of Physics: Condensed Matter 14 R169-R193

Rampe, A., Hartmann, D., Weber, W., Popovic, S., Reese, M., and Güntherodt, G., (1995) Induced spin polarization and interlayer exchange coupling of the system $\mathrm{Rh} / \mathrm{Co}$ (0001) and Ru/Co (0001) Physical Review B 51, 3230-3233

Rehbein A., Wegner D., Kaindl G. and Bauer A, (2003), Temperature dependence of lifetimes of Gd(0001) surface states, Physical Review B 67, 033403-033406

Rotenberg, Eli, Wu, Y.Z., An, J.M., Van Hove, M.A., Canning, A., Wang, L.W. and Qiu, Z.Q., (2006) Non-free-electron momentum- and thickness-dependent evolution of quantum well states in the $\mathrm{Cu} / \mathrm{Co} / \mathrm{Cu}(001)$ system, Physical Reiew. B 73, 075426-075429 Roy, D. and Carette, J.D., (1977) Electron Spectroscopy for Surface Analysis, H Ibach (Ed.) Springer, Berlin p 13 
Samant, M.G., Stöhr, J., Parkin, S.S.P., Held, G.A., Hermsmeier, B.D., and Herman, F., (1994) Induced spin polarization in $\mathrm{Cu}$ spacer layers in $\mathrm{Co} / \mathrm{Cu}$ multilayers Physical Review Letters 72 1112-1115

Scheinfein, M.R., Pierce, D.T., Unguris, J., McClelland, J.J., Celotta, R.J. and Kelley, M.H., (1989) Improved low-energy diffuse scattering electron-spin polarization analyzer Review of Scientific Instruments 60, 1-11

Schönhense, G. and Siegmann, H.C., (1993) Transmission of Electrons through Ferromagnetic Material and Applications to Detection of Electron-Spin Polarization Annalen der Physik, Leipzig, 2 465-474

Siegmann, H.C., (1994) Surface and 2D magnetism with spin polarized cascade electrons Surface Science 307-309, 1076-1086

Sinkovic, B., Shekel, E. and Hulbert, S.L., (1995) Spin-resolved Fe $L_{3} M_{45} M_{45}$ Auger transition on and off resonance: The effect of exchange correlation Physical Review B, 52, R15703-R15706

Schwarz K., (1986) $\mathrm{CrO}_{2}$ predicted as a half-metallic ferromagnet Journal of Physics F:

Metal Physics 16, L211-L215

Sinkovic, B., Tjeng, L.H., Brookes, N.B., Goedkoop, J.B., Hesper, R., Pellegrin, E., de Groot, F.M.F., Altieri, S., Hulbert, S.L., Shekel, E., and Sawatzky, G.A., (1997) Local Electronic and Magnetic Structure of $\mathrm{Ni}$ below and above $\mathrm{T}_{\mathrm{c}}$ : A Spin-Resolved Circularly Polarized Resonant Photoemission Study Physical Review Letters 79, 35103513

Smith, N.V., Brookes, N.B., Chang, Y. and Johnson, P.D., (1994) Quantum-well and tight-binding analyses of spin-polarized photoemission from $\mathrm{Ag} / \mathrm{Fe}(001)$ overlayers 
Physical Review B 49, 332-338

Soulen, Jr., R. J., Byers, J. M., Osofsky, M. S., Nadgorny, B., Ambrose, T., Cheng, S. F., Broussard, P.R., Tanaka, C. T., Nowak, J., Moodera, J. S., Barry, A., and Coey, J. M. D., (1998) Measuring the Spin Polarization of a Metal with a Superconducting Point Contact Science 282, 85-88

Stanka, B., Hebenstreit, W., Diebold, U., and Chambers, S.A., (2000) Surface reconstruction of $\mathrm{Fe}_{3} \mathrm{O}_{4}(001)$ Surface Science 448, 49-63

Steeneken, P.G., Tjeng, L.H., Elfimov, I., Sawatzky, G.A., Ghiringhelli, G., Brookes, N.B., and Huang, D.-J., (2002) Exchange Splitting and Charge Carrier Spin Polarization in EuO Physical Review Letters 88, 047201-047204

Suga, S., et al. (2006), to be published

Tang, F.C., Zhang, X., Dunning, F.B. and Walters, G.K., (1988) Compact low-energy Mott polarimeter for use in energy- and angle-resolved polarization studies Review of Scientific Instruments 59, 504-505

Tarrach, G., Bürgler, D., Schaub, T., Wiesendanger, R., Güntherodt, H.-J., (1993) Atomic surface structure of $\mathrm{Fe}_{3} \mathrm{O}_{4}(001)$ in different preparation stages studied by scanning tunneling microscopy Surface Science 285, 1-14

De Teresa, J.M., Barthélémy, A., Fert, A., Contour, J.P., Lyonnet, R., Montaigne, F., Seneor, P. and VaurЭs, A., (1999) Inverse Tunnel Magnetoresistance in $\mathrm{Co} / \mathrm{SrTiO}_{3} / \mathrm{La}_{0.7} \mathrm{Sr}_{0.3} \mathrm{MnO}_{3}$ : New Ideas on Spin-Polarized Tunneling Physical Review Letters 82, 4288-4291

Tillman, D., Thiel, R. and Kisker, E., (1989) (Rapid Note) Very-low-energy spinpolarized electron diffraction from Fe(001) Zeitschrift Physik B 77, 1-2 
Tjeng, L.H., Brookes, N.B., Goedkoop, J.B., Sinkovic, B., de Groot, F.M.F., Hesper, R., Altieri, S., Pellegrin, E., Tanaka, A., Hulbert, S.L., Shekel, E., Hien, N.T., Menovsky, A.A., and Sawatzky, G.A., (1999) Study of Magnetic Materials Using Spin-Resolved Circularly-Polarized Resonant Photoemission Japan Journal of Applied Physics 38, Supplement 38-1, 344-347

Tjeng, L.H., Sinkovic, B., Brookes, N.B., Goedkoop, J.B., Hesper, R., Pellegrin, E., de Groot, F.M.F., Altieri, S., Hulbert, S.L., Shekel, E., and Sawatzky, G.A., (1997) SpinResolved Photoemission on Anti-Ferromagnets: Direct Observation of Zhang-Rice Singlets in $\mathrm{CuO}$ Physical Review Letters 78, 1126-1129

Tjeng, L.H., Brookes, N.B., and Sinkovic, B., (2001) Spin-resolved photoelectron spectroscopy on cuprate systems Journal of Electron Spectroscopy and Related Phenomena 117-118, 189-201

Tsymbal, E.Yu., and Pettifor, D.G. (1997). J. Phys.: Condens. Matter 9, L411.

Unguris, J., Pierce, D.T. and Celotta, R.J., (1986) Low-energy diffuse scattering electronspin polarization analyzer Review of Scientific Instruments 57, 1314-1323

Van der Sluijs, A., Drouhin, H-J., Lampel, G., Lassailly, Y. and Marliere, C.R., (1994) Spin-dependent transmission through ultra-thin magnetic layers - towards highly discriminative compact spin detectors, Comptes Rendus De L'Academie des Sciences, Paris 319 Series II 753-759

Van Gelderen, P., Crampin, S., and Inglesfield, J.E., (1996) Quantum-well states in Cu/Co overlayers and sandwiches Physical Review B $539115-9122$ 
Vescovo, E., Carbone, C., Eberhardt, W., Rader, O., Kachel, T. and Gudat, W. (1993)

Spin-resolved photoemission study of the clean and oxygen-covered $\mathrm{Fe}(110)$ surface Physical Review B 48 285-288

Weber, W., Wesner, D.A., Güntherodt, G., and Linke, U., (1991) Direct Observation of Spin-Split Electronic States of Pd at the Pd(111)/Fe(110) Interface Physical Review Letters 66, 942-945

Weber, W., Wesner, D.A., Hartmann, D., and Güntherodt, G., (1992) Spin-polarized interface states at the $\mathrm{Pd}(111) / \mathrm{Fe}(110), \operatorname{Pd}(111) / \mathrm{Co}(0001)$, and $\operatorname{Pt}(111) / \operatorname{Co}(0001)$ interfaces Physical Review B 46, 6199-6206

Weber, W., Wesner, D.A., Hartmann, D., Effner, U.A., and Güntherodt, G., (1993) Electronic interface states at the Pt/Co interface Journal of Magnetism and Magnetic Materials 121, 156-159

Weschke, E., Schussler- Langeheine, C., Meier, R., Fedorov, A.V., Starke, K., Hubinger, F. and Kaindl, G., (1996) Physical Review Letters 77, 3415-3418

Wu, R., Li, C., Freeman, A.J. and Fu, C.L., (1991) Structural, electronic, and magnetic properties of rare-earth metal surfaces: hcp Gd(0001) Physical Review B 44, 9400-9409 Wu, Y.Z., Won, C., Rotenberg, E., Zhao, H.W., Xue, Qi-Kun, Kim, W., Owens, T.L., Smith, N.V., and Qiu, Z.Q., (2006) Resonant interaction between two Cu quantum wells, Physical Review B, in press.

Wurmehl S., Fecher, G.H., Kandpal, H.C., Ksenofontov, V., Felser, C., Lin, H.-J., and Morais, J., (2005) Geometric, electronic, and magnetic structure of $\mathrm{Co}_{2} \mathrm{FeSi}$ : Curie temperature and magnetic moment measurements and calculations Physical Review B 72, $184434-184442$ 
Yanese, A., and Siratori, K., (1984) Band Structure in the High Temperature Phase of $\mathrm{Fe}_{3} \mathrm{O}_{4}$ Journal of the Physical Society of Japan 53, 312-317

Yuasa, S., Nagahama, T., Fukushima, A., Suzuki, Y., and Ando, K., (2004) Giant roomtemperature magnetoresistance in single-crystal $\mathrm{Fe} / \mathrm{MgO} / \mathrm{Fe}$ magnetic tunnel junctions.

Nature Materials 3, 868-871

Yuasa, S., Fukushima, A., Kubota, H., Suzuki, Y., and Ando, K., (2006) Giant tunneling magnetoresistance up to $410 \%$ at room temperature in fully epitaxial $\mathrm{Co} / \mathrm{MgO} / \mathrm{Co}$ magnetic tunnel junctions with bcc $\mathrm{Co}(001)$ electrodes Applied Physics Letters 89, 042505-042507

Zener, C., (1951) Interaction Between the $d$ Shells in the Transition Metals Physical Review 81, 440-444; Zener, C., (1951) Interaction between the $d$-Shells in the Transition Metals. II. Ferromagnetic Compounds of Manganese with Perovskite Structure Physical Review 82, 403-405; Zener, C., (1951) Interaction between the $d$-Shells in the Transition Metals. III. Calculation of the Weiss Factors in Fe, Co, and Ni Physical Review 83, 299301

Zhang, F. C., and Rice, T. M. (1988) Effective Hamiltonian for the superconducting $\mathrm{Cu}$ oxides Physical Review B 37, 3759-3761

Zhang, Z., and S. Satpathy, (1991) Electron states, magnetism, and the Verwey transition in magnetite Physical Review B 44, 13319-13331

Zhang, Z.D., Choi, H.J., Kawakami, R.K., Escorcia-Apericio, Enresto, Bowen, Martin Wolfe, Jason, Rotenberg, E., Smith, N.V., and Qiu, Z.Q., (2000) Magnetic interlayer coupling between $\mathrm{Co}$ films across $\mathrm{Cu} / \mathrm{Ni}_{30} \mathrm{Cu}_{70} / \mathrm{Cu}(100)$ double quantum wells, Physical Review B 61, 76-79 
Zhang, X.-G., Butler, W.H., and Bandyopadhyay, A., (2003) Effects of the iron-oxide layer in Fe-FeO-MgO-Fe tunneling junctions Physical Review B 68, 092402-092405

Zydb, R. and Bauer, E., (2002) Spin-resolved unoccupied electronic band structure from quantum size oscillations in the reflectivity of slow electrons from ultrathin ferromagnetic crystals Physical Review Letters 88, 166403-166406 


\section{Figure Captions:-}

Figure 1. A schematic of the photoemission process. The incident photon with energy $\hbar \omega$ excites an electron from an initial state below the Fermi level $E_{\mathrm{F}}$ to some final state above the vacuum level $E_{\mathrm{vac}}$. The left panel shows the electron originating either from the valence band or the more localized core level. The right panel displays the excited electron energy distribution in the final state.

Figure 2. A schematic of the direct k-conserving transition in the photoemission process. The incident photon excites an electron from an initial state $\mathrm{E}_{\mathrm{i}}$ below the Fermi level $\mathrm{E}_{\mathrm{F}}$ to some final state $\mathrm{E}_{\mathrm{f}}$ above the vacuum level $\mathrm{E}_{\mathrm{V}}$. Spin conservation is maintained in the transition.

Figure 3. Overview of a spin-resolved spectrometer system. A Mott spin polarimeter is coupled to a hemispherical electron energy analyzer via an input lens. An electron multiplier array assembly located in the exit plane of the energy analyzer is used for non-spin-resolved multichannel detection.

Figure 4. Cross-sectional view of the Mott spin polarimeter with enlarged conic-type collection angle. Only one pair of electron multipliers is shown; the other pair is located in the perpendicular plane. 
Figure 5. Total scattering cross section $\sigma=1 / \lambda \mathrm{in} \mathrm{nm}^{-1}$ against the number of unoccupied d-orbitals for the transition metals indicated. The data are obtained by analysing the electron spin polarization in overlayer films and are valid for electron energies within $5 \mathrm{eV}$ of the vacuum level.

Figure 6. Experimentally determined $E$ vs $k$ dispersion for the surface state at the center of the zone on a $\mathrm{Au}(111)$ surface. The measured data points are indicated by the open circles and filled triangles; the solid and dashed lines are parabolic fits. The fit results are $\left(\mathrm{eV}\right.$ and $\left.\AA^{-1}\right): E_{1}(\mathrm{k})=15.2(k-0.0117)^{2}-0.416$, and $E_{2}(k)=15.3(k+0.0117)^{2}-0.418$. (From La Shell et al., 1996)

Figure 7. Spin-resolved photoemission spectra from the Au(111) surface state at various polar emission angles. The photon energy was $\hbar \omega=21.1 \mathrm{eV}$. The spin assignment was determined from the transverse in-plane component of the polarization vector. Spin-up and spin-down intensities are marked with filled and open triangles, respectively. (From Hoesch et al., 2004)

Figure 8. Graphical solutions (full circles) for the energies of quantum well states of Ag overlayers on $\mathrm{Fe}(001)$ using the phase accumulation model. Full bold waves represent the phase $2 \pi n-\varphi_{\mathrm{C}}-\varphi_{\mathrm{B}}$ and dashed curves represent the quantum well phase accumulation $m 2 k a$. States characterized by the quantum number $v=(m-n)$ are connected by the full thin curves. (From Smith et al., 2004) 
Figure 9. Spin-integrated photoemission spectra recorded from silver films deposited on an $\mathrm{Fe}(001)$ substrate. The different film thicknesses are indicated.

Figure 10. Energy bands of $\mathrm{Fe}$ and $\mathrm{Ag}$ along the $\Delta$ direction for both minority and majority spins. The toned areas indicate the range of the s-d hybridization gap in Fe for states of $\Delta_{1}$ symmetry.

Figure 11. (a) Oscillations in the inverse photoemission intensity at $E_{\mathrm{F}}$ for copper films deposited on a fcc $\operatorname{Co}(001)$ substrate from the study of Ortega et al (1993). (b) Results of a Kerr effect study showing the oscillatory antifferomagnetic coupling in $\mathrm{Cu} / \mathrm{Co}(001)$ multilayers as a function of the copper thickness from the study of Qiu et al (1992).

Figure 12. Spin-resolved photoemission spectra recorded from two, six and eight monolayer thick copper films deposited on a fcc $\mathrm{Co}(001)$ substrate. The minority and majority spin spectra are represented by the open and full diamonds, respectively. The spectra are recorded for photoelectrons emitted along the surface normal.

Figure 13 Spin-resolved photoemission spectra recorded from the $\operatorname{Gd}(0001)$ surface at $20 \mathrm{~K}$. The upper and lower spectra represent the emission in the majority- and minorityspin channels, respectively. The lines indicate Lorentzian fits to the spectra superimposed on appropriate backgrounds. The inset shows the relative intensities in the two spin channels. 
Figure 14 Left panel: Spin-resolved photoemission intensities ( $\Delta$ spin up, $\nabla$ spin down) measured at $\mathrm{h} v=21.2 \mathrm{eV}$ together with the total intensity $(\bullet)$ of $5 \mathrm{~nm}$ thick Fe(110) (bottom) and $\mathrm{Fe}_{3} \mathrm{O}_{4}(111)$ (top), obtained by oxidizing $\mathrm{Fe}(110)$ in oxygen atmosphere at $250^{\circ}$ C. Right panel: Spin polarization of $\mathrm{Fe}(110)(\square)$ and $\mathrm{Fe}_{3} \mathrm{O}_{4}(111)(\bullet)$. (Dedkov et al., 2002a)

Figure 15. Right panel: Spin polarization as a function of binding energy of an epitaxial $\mathrm{CrO} 2(100)$ film (a) after $\mathrm{Ar}^{+}$sputtering for $210 \mathrm{~s}$ at $500 \mathrm{eV}$ and (b) after $750 \mathrm{~s}$ sputtering and an additional annealing treatment at $150{ }^{\circ} \mathrm{C}$ for $12 \mathrm{~h}$. Left panel: Corresponding spinpolarized photoemission spectra at $\mathrm{h} v=21.2 \mathrm{eV}(\nabla$ spin down, $\boldsymbol{\Delta}$ spin up $)$ and total photoemission intensity (•). (Dedkov et al., 2002b)

Figure 16. Temperature dependence of spin polarization of Mn $3 d$ states, $e_{g}$ at $1.0 \mathrm{eV}$ binding energy and $t_{2 g}$ at $2.2 \mathrm{eV}$ binding energy obtained with $h \mathrm{y}=40 \mathrm{eV}$. The inset shows the spin-polarized photoemission spectra for the majority and minority spins and the difference at $40 \mathrm{~K}$; after (Park et al., 1998)

Figure 17 Left panel: The spin-resolved photoemission spectra ( $\boldsymbol{\nabla}$ spin down, $\Delta$ spin up) together with the total emission intensity (•) of $\mathrm{Fe}(110), 2 \AA \mathrm{MgO}$ on $\mathrm{Fe}(110)$, and $5 \AA$ $\mathrm{MgO}$ on $\mathrm{Fe}(110)$ (from bottom to top). Right panel: The spin polarization as function of binding energy of a 50- $\AA$ thick $\mathrm{Fe}(110)$ film (匹), $2 \AA \mathrm{MgO}$ on $\mathrm{Fe}(110)(\mathrm{O})$, and $5 \AA$ $\mathrm{MgO}$ on $\mathrm{Fe}(110)(\bullet)$. (Dedkov et al., 2006) 
Figure 18. The change of the normalized spin polarization at $E_{\mathrm{F}}$ of the $\mathrm{MgO} / \mathrm{Fe}(110)$ system with increasing $\mathrm{MgO}$ layer thickness. The spline fit to the experimental data is shown by a solid line. The reference curves for depolarization of polarized electrons $\left(P_{0}\right.$ $=23.5 \%$ ) optically excited in germanium after traversing an evaporated overlayer of $\mathrm{Ni}$ (dashed line), Gd (dotted line), and Ce (dot-dashed line) are taken from (Meier et al., 1984). (Dedkov et al., 2006).

Figure 19 (a) The spin-resolved photoemission spectra ( $\boldsymbol{\nabla}$ spin down, $\Delta$ spin up ) together with the total emission intensity $(\bullet)$ for $\mathrm{W}(110), \mathrm{Fe}(110), 7 \AA \mathrm{Al}$ on $\mathrm{Fe}(110)$, and approximately $1 \mathrm{ML} \mathrm{Al}_{2} \mathrm{O}_{3}$ on $\mathrm{Fe}(110)$. (b) The spin polarization as function of binding energy of a 50-Å-thick $\mathrm{Fe}(110)$ film (घ), $7 \AA \AA \mathrm{Al}$ on $\mathrm{Fe}(110)(\boldsymbol{\Delta})$, and approximately 1 $\mathrm{ML} \alpha-\mathrm{Al}_{2} \mathrm{O}_{3}$ on $\mathrm{Fe}(110)(\bullet)$. (Dedkov et al., 2002c).

Figure 20. Spin-polarized photoemission spectra from $\mathrm{Bi}_{2} \mathrm{Sr}_{2} \mathrm{CaCu}_{2} \mathrm{O}_{8+\delta}$. (a) The spinintegrated resonant photoemission spectra taken at the $\mathrm{Cu} L 3$ absorption edge (full line). The symbols show the integrated spectra separated into its singlet ( $\mathbf{\square})$ and triplet $(\boldsymbol{\Delta})$ components. (b) The measured spin polarization corresponding to the spectra in panel (a). 
Figure 1.

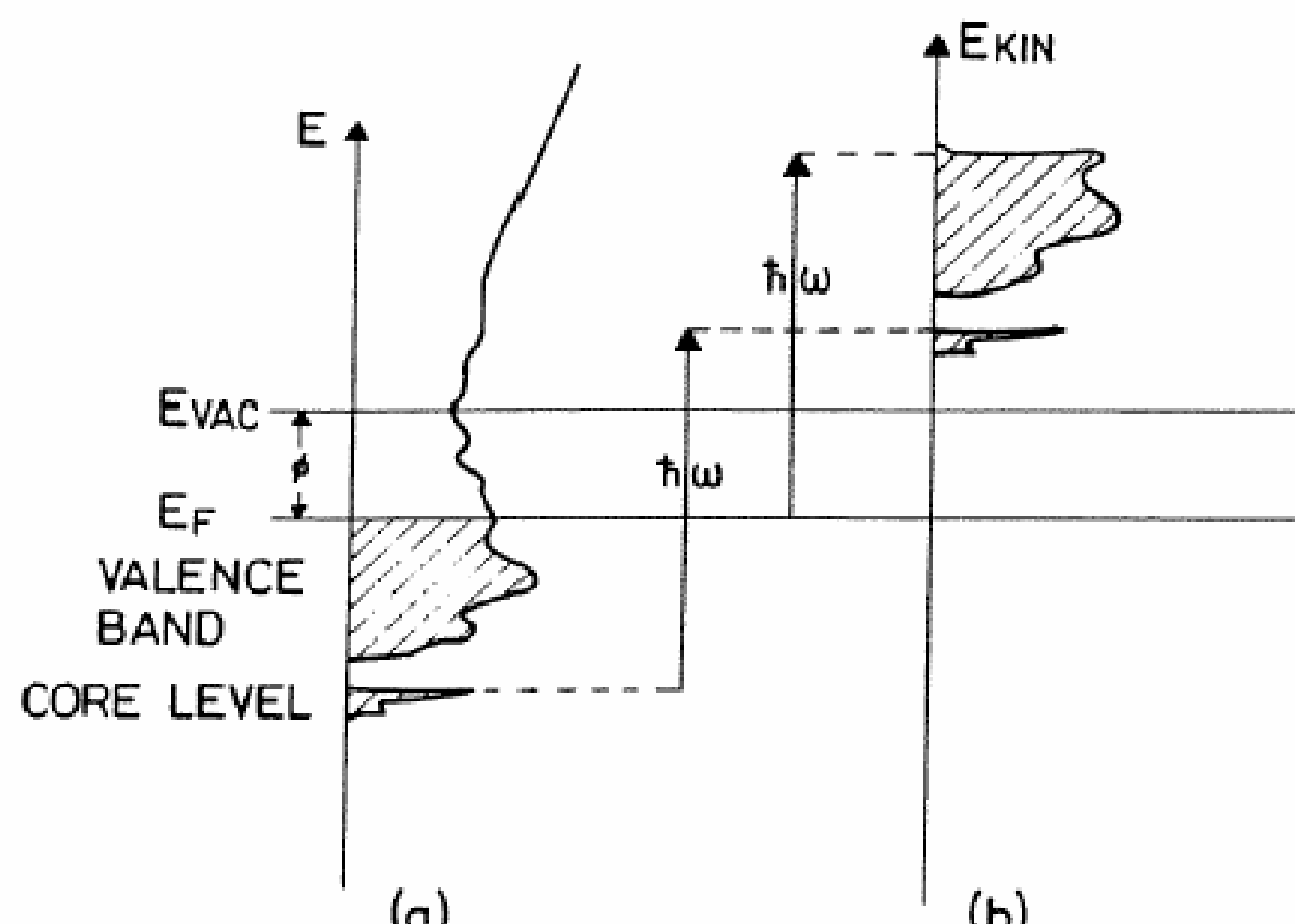

(a)

(b) 
Figure 2.

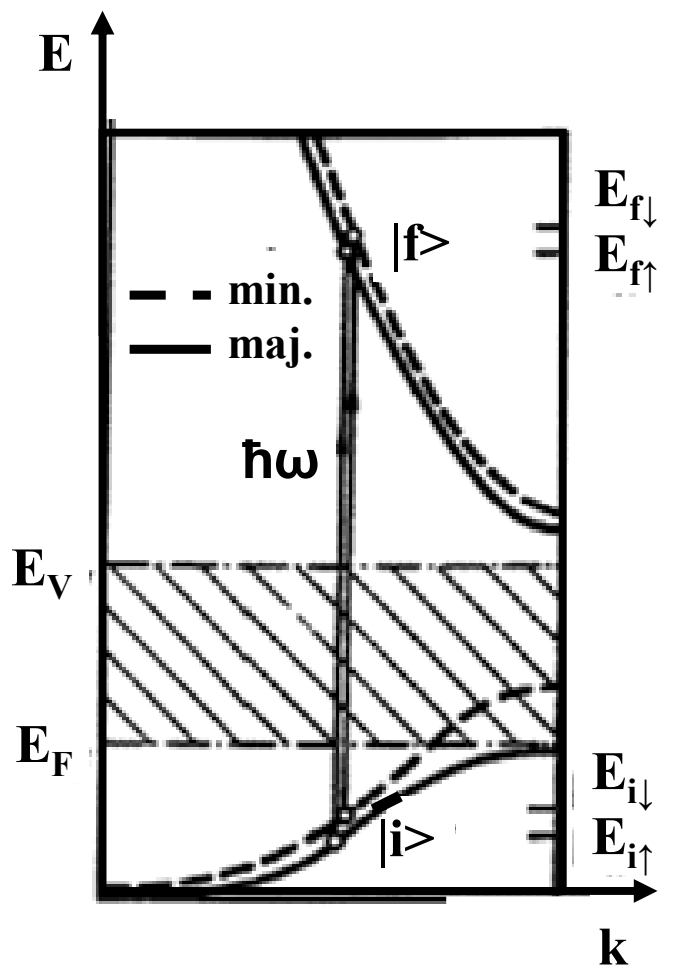


Figure 3.

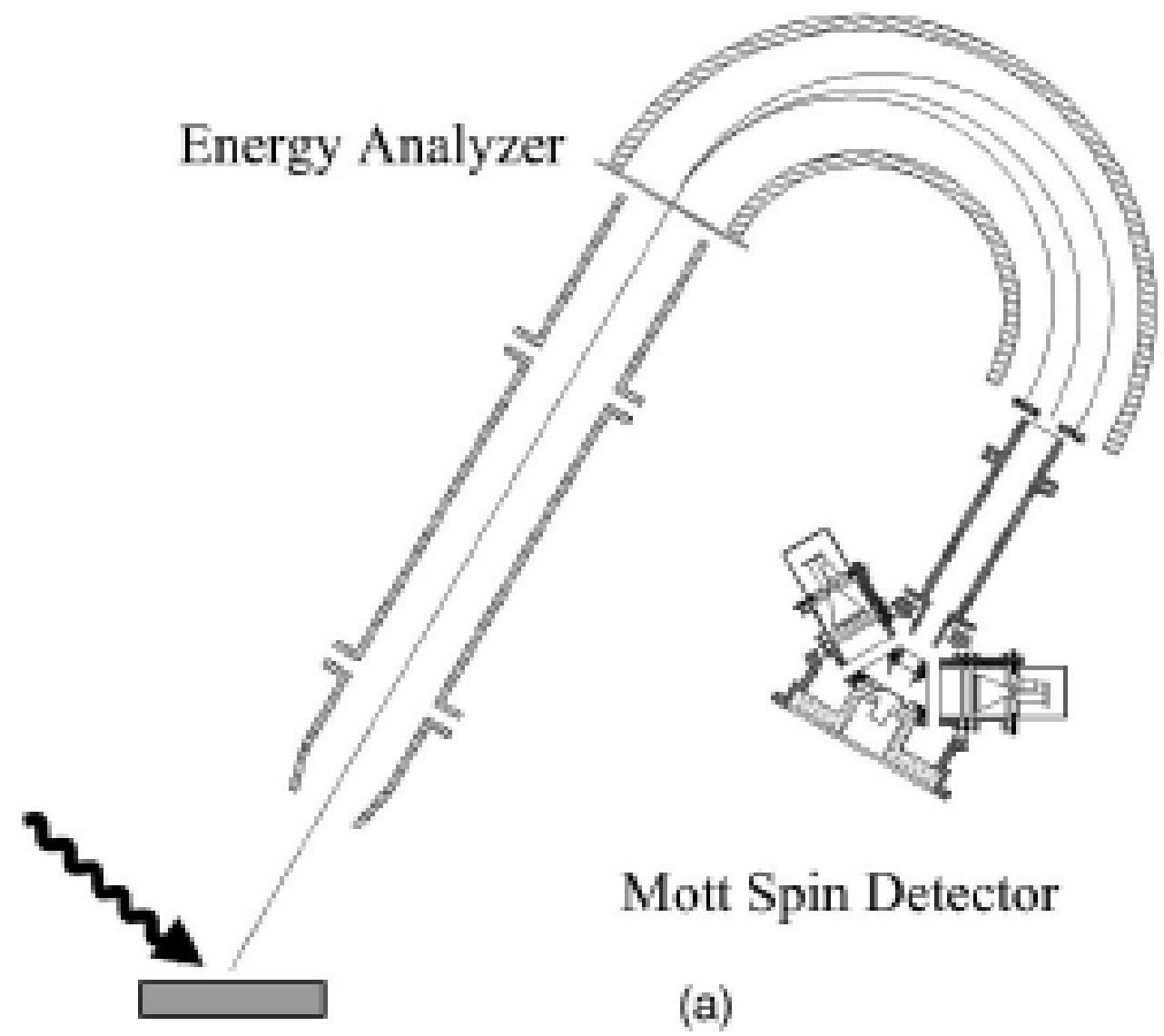


Figure 4.

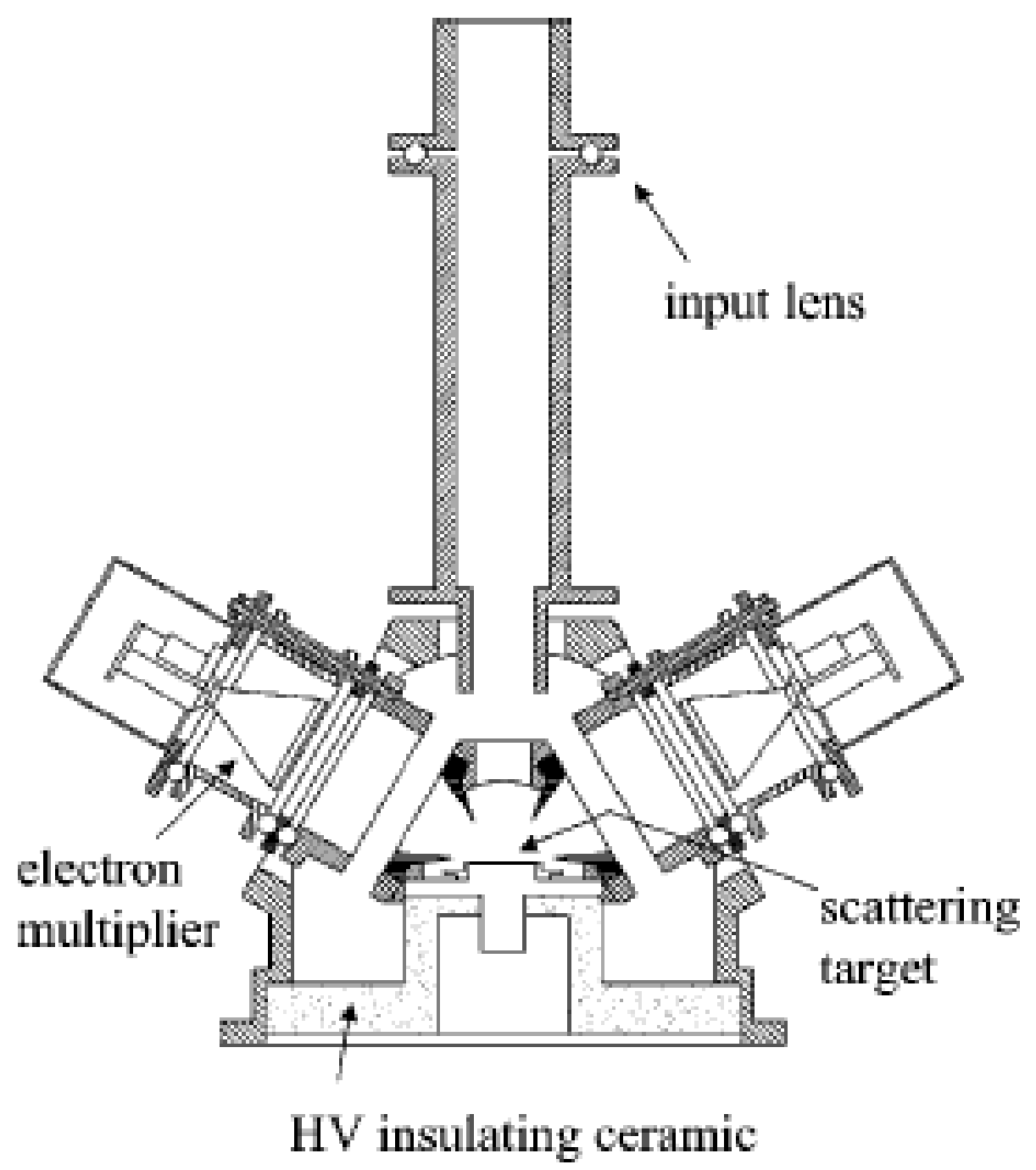


Figure 5.

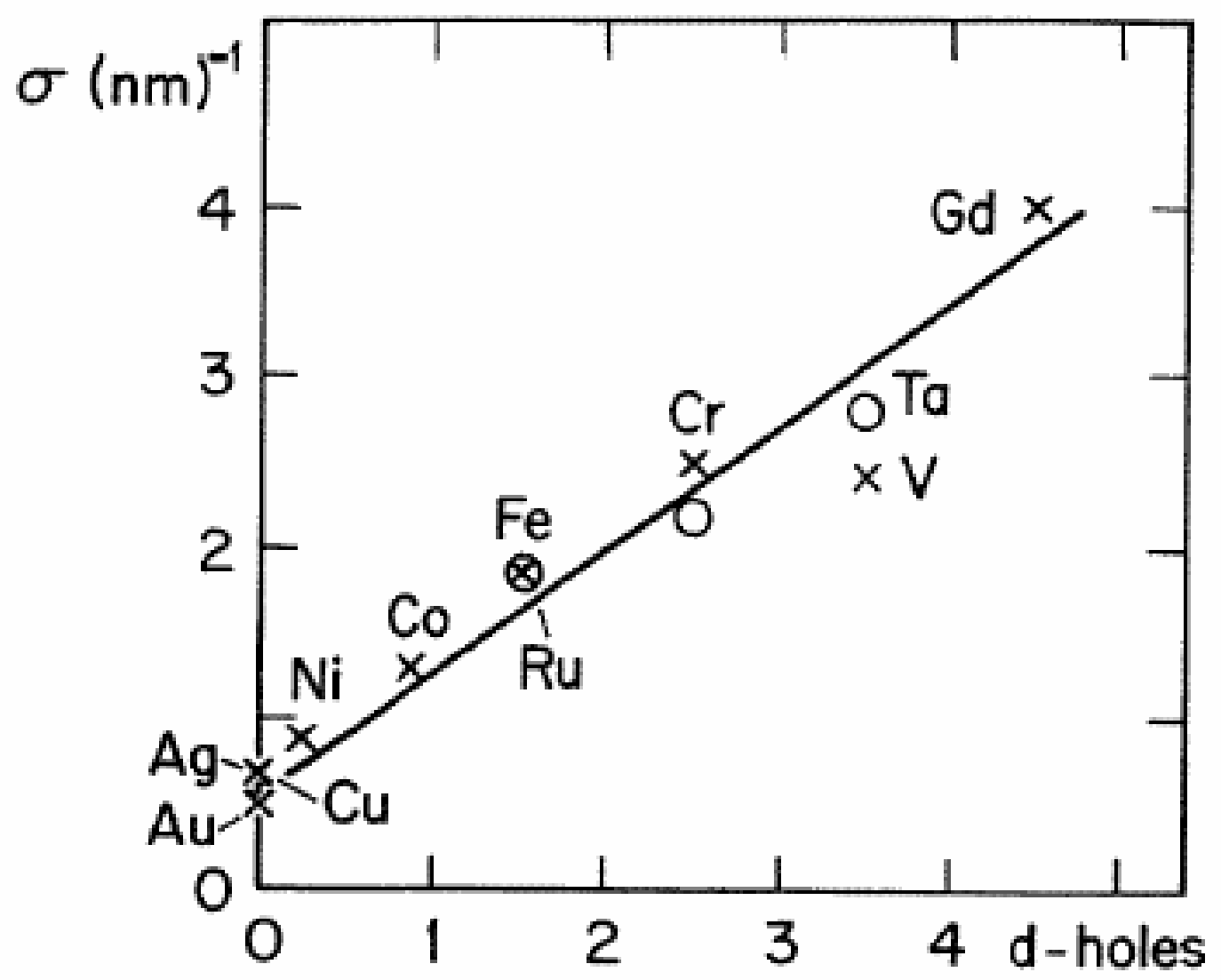


Figure 6.

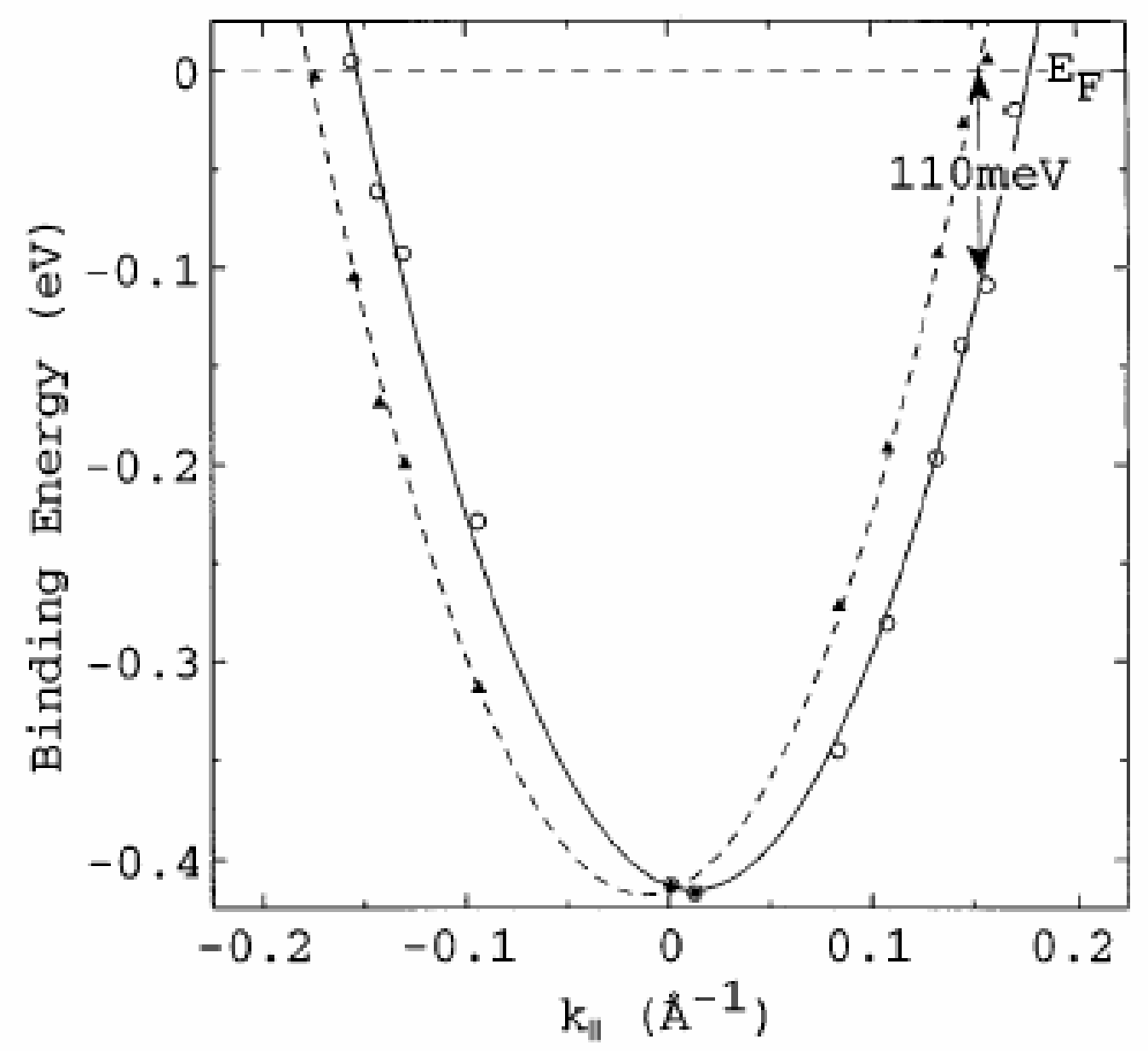


Figure 7.

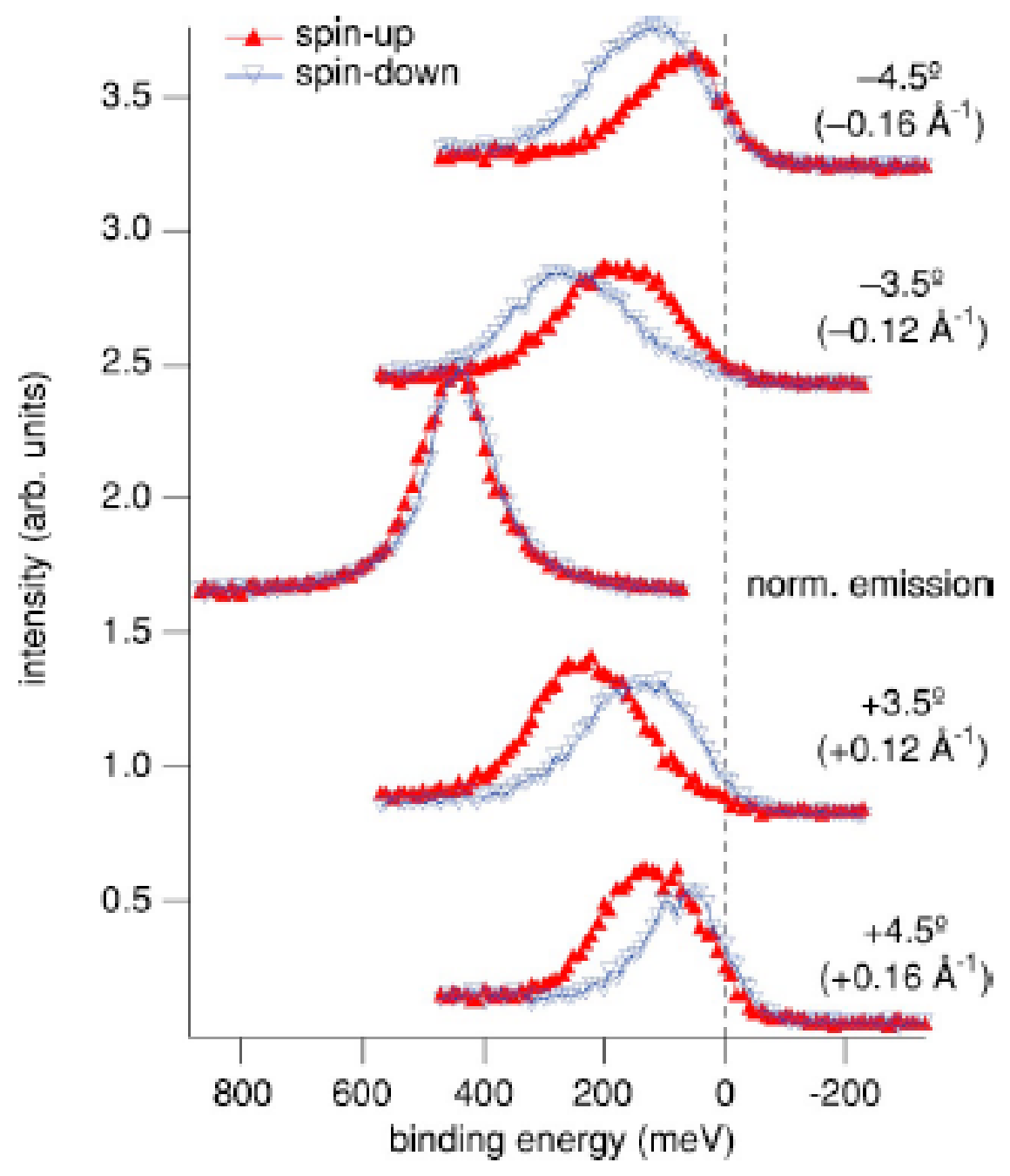


Figure 8.

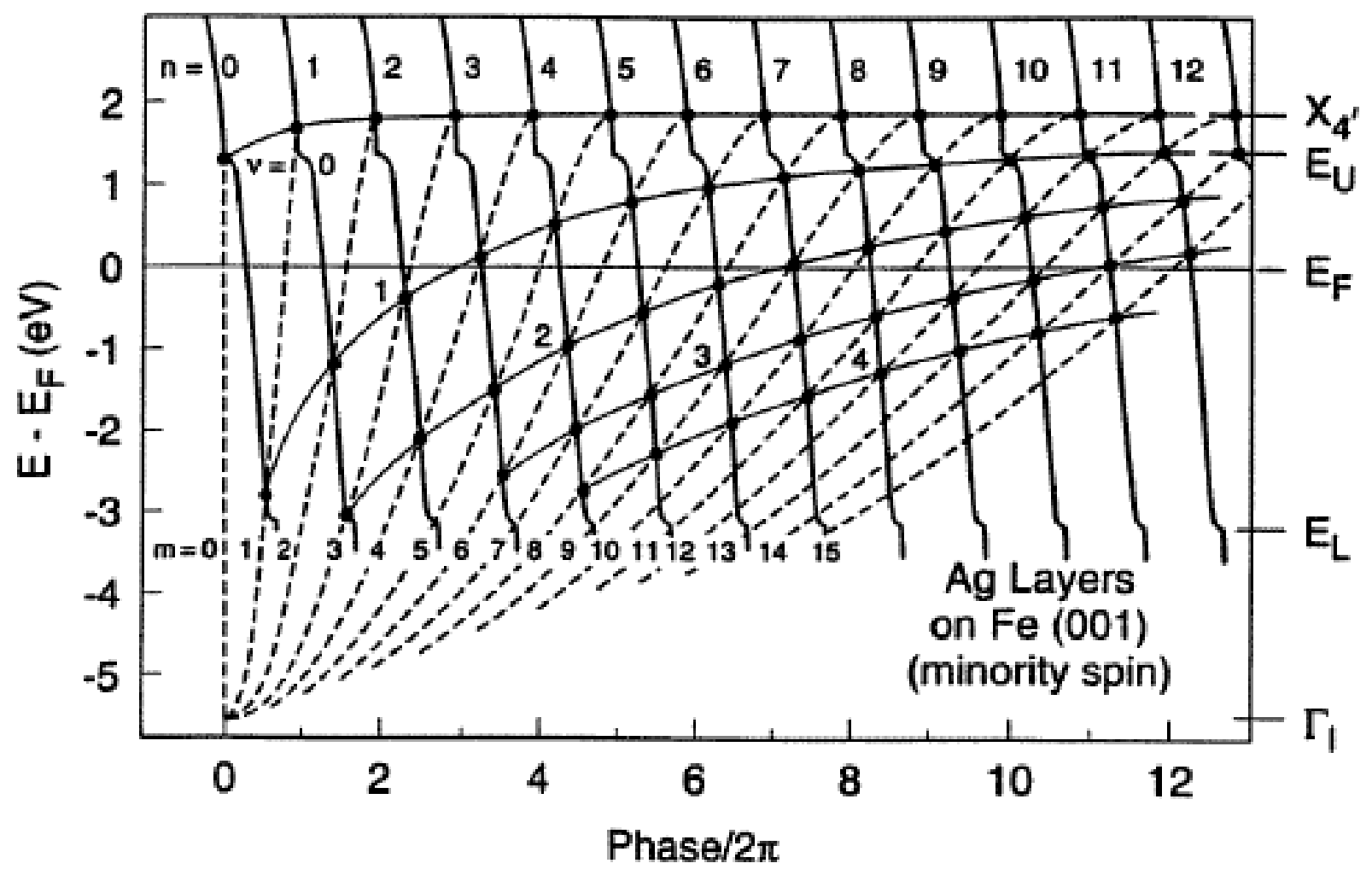


Figure 9.

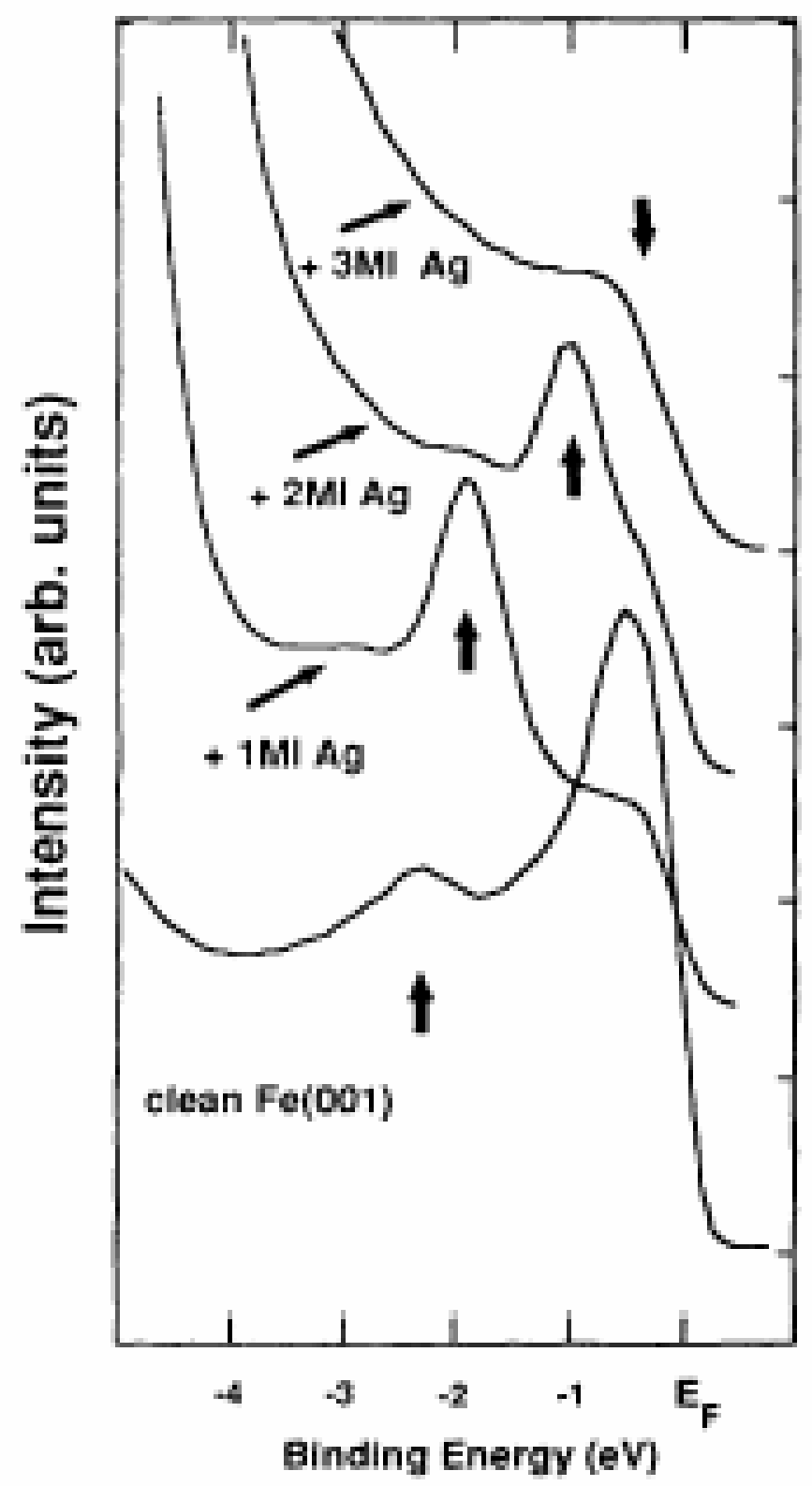


Figure 10.
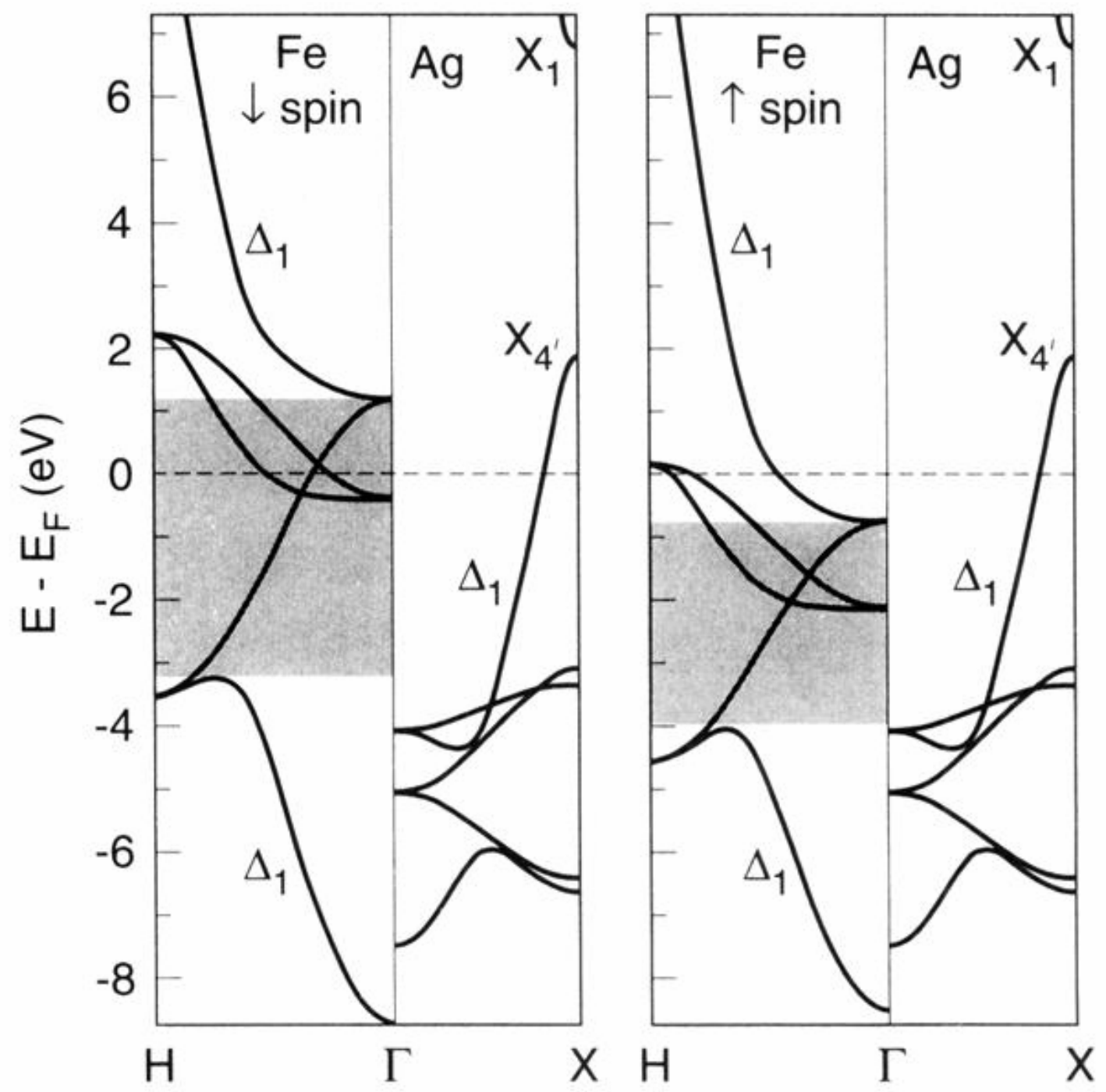
Figure 11.

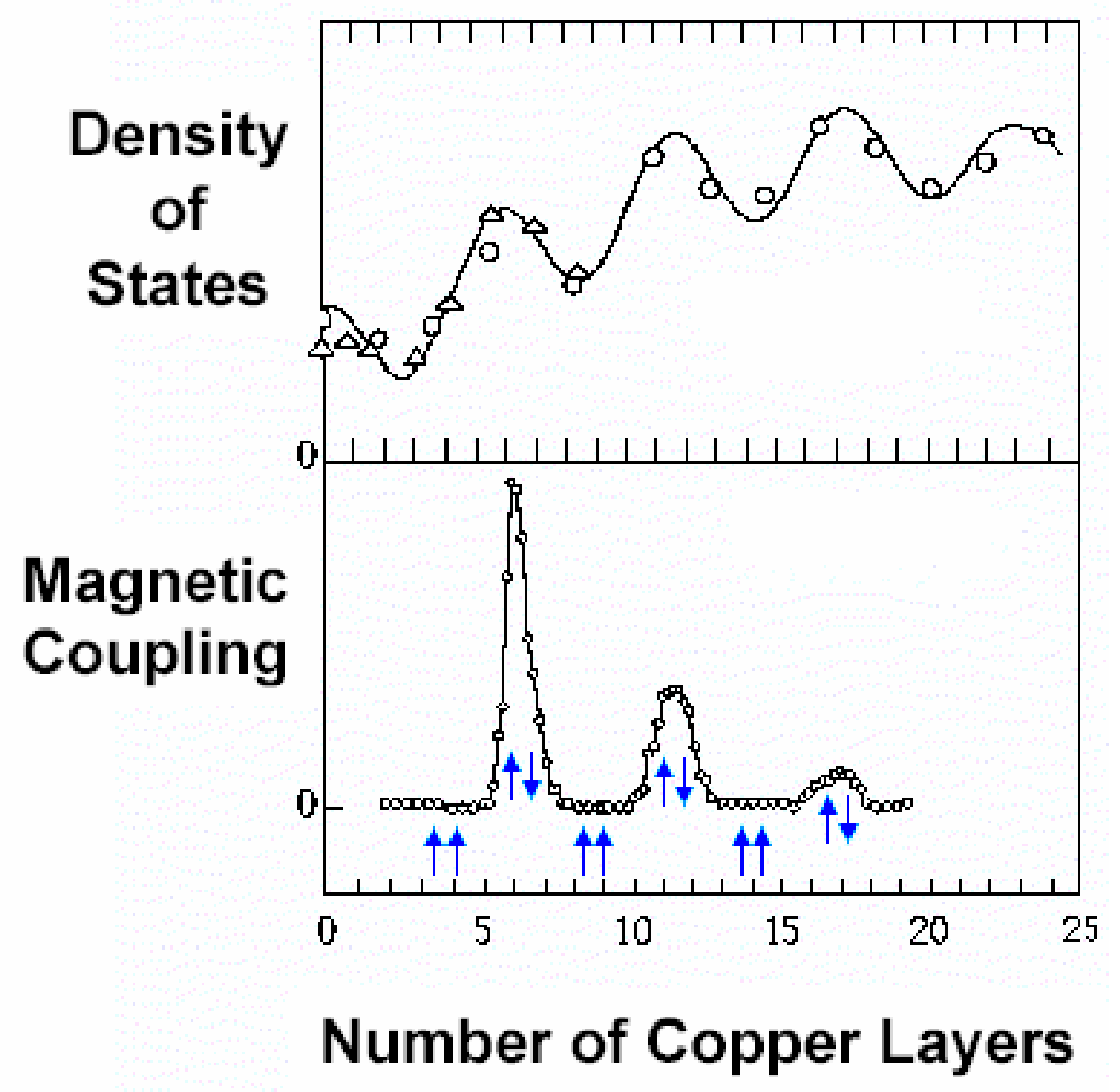


Figure 12.

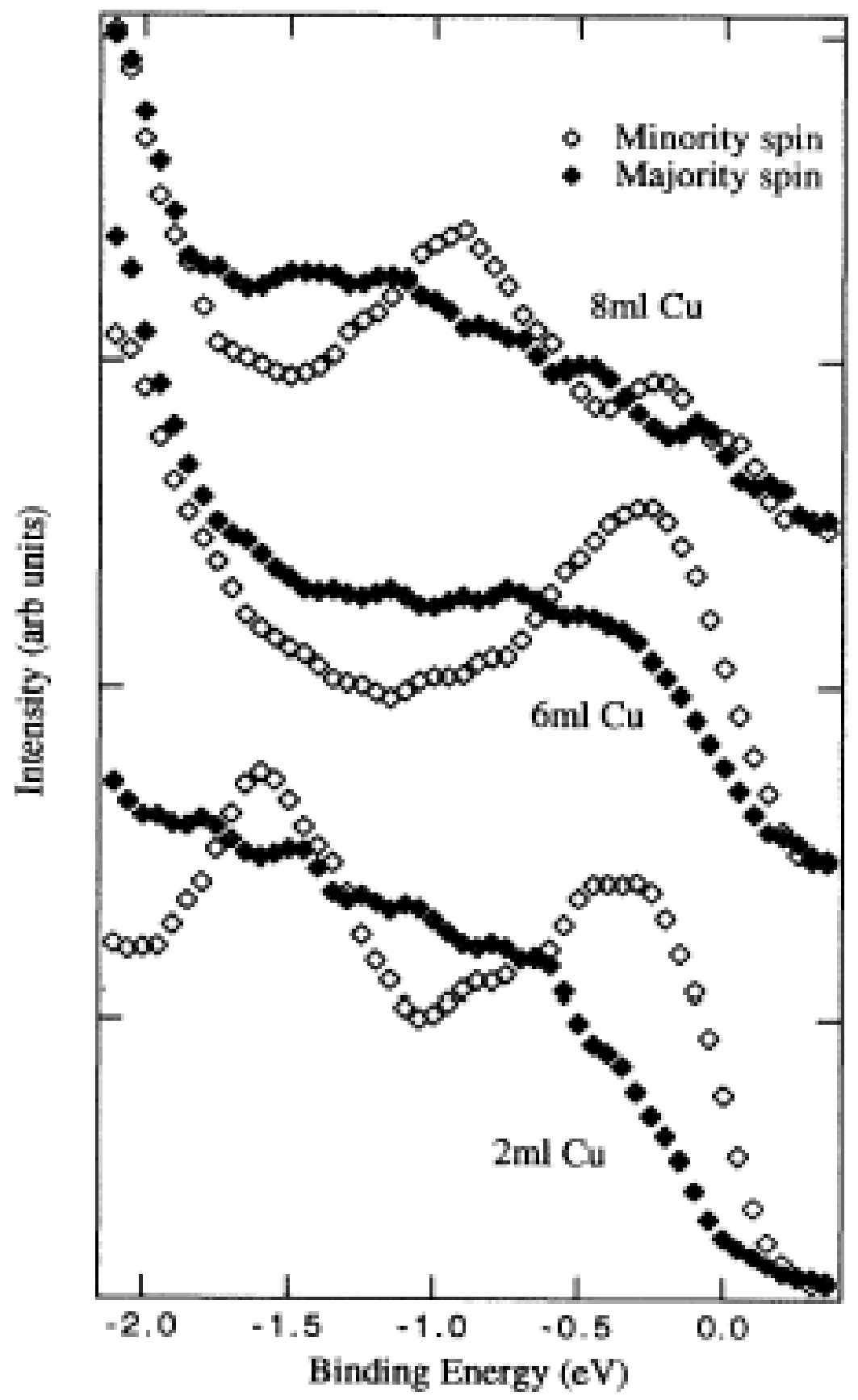


Figure 13.

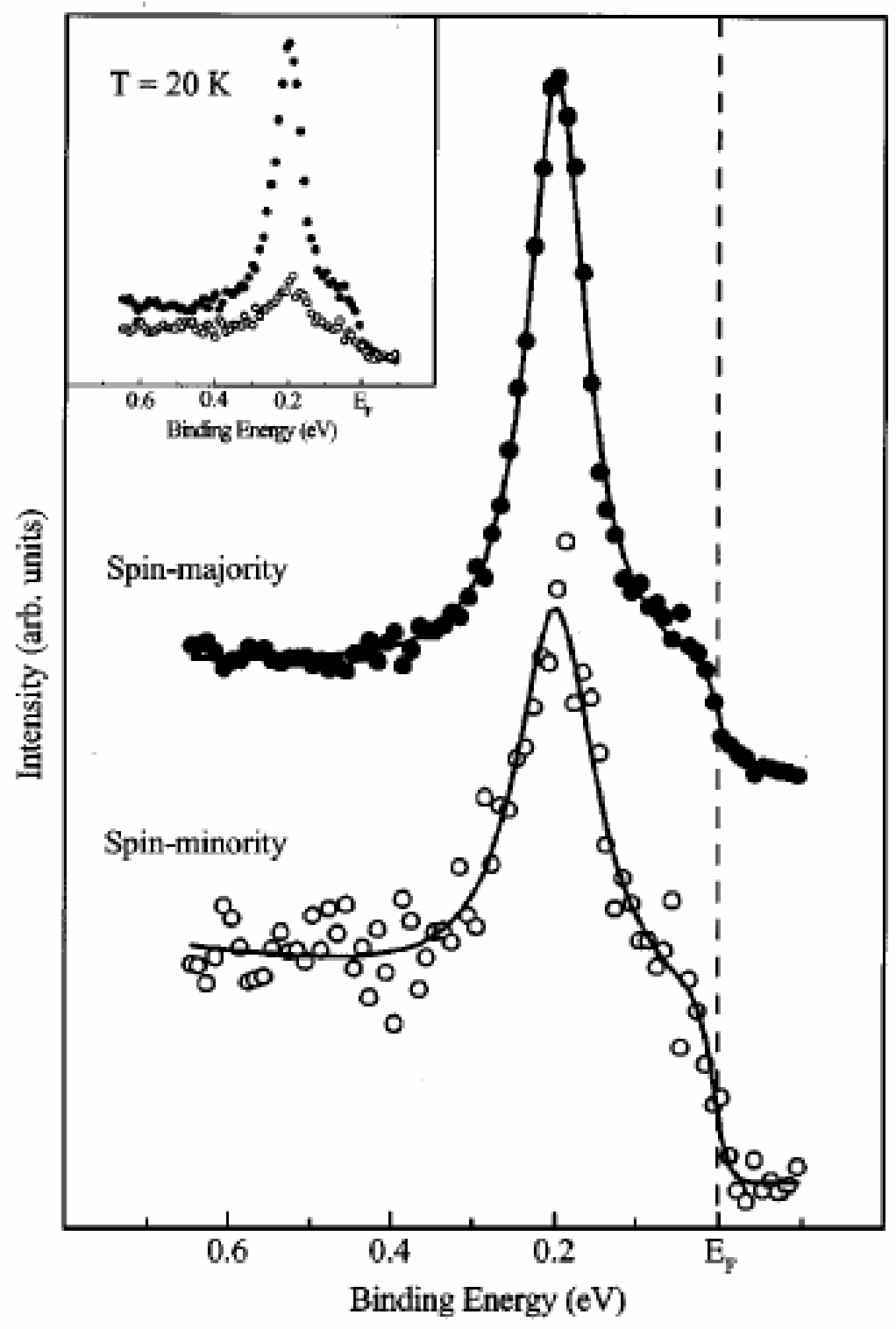


Figure 20.

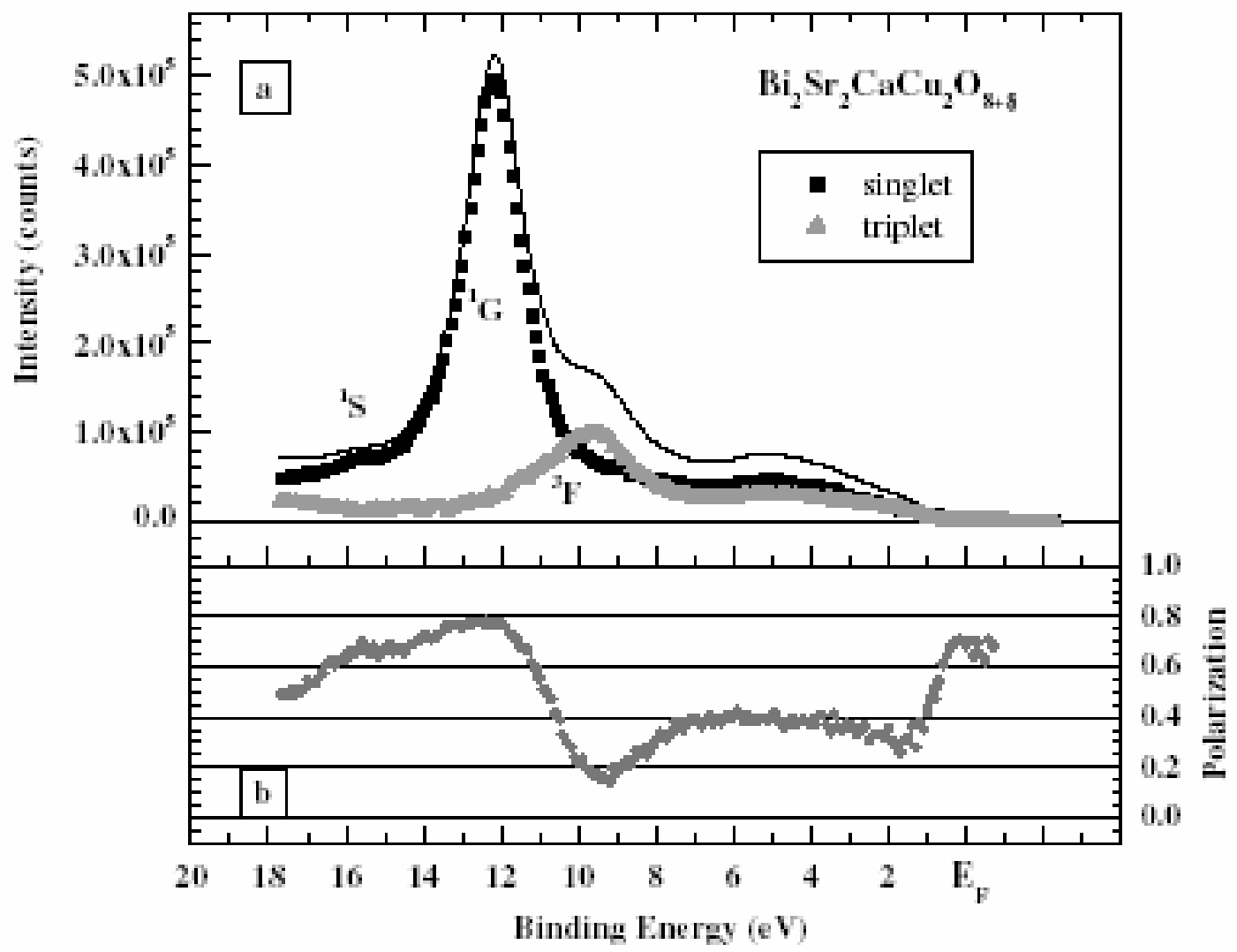









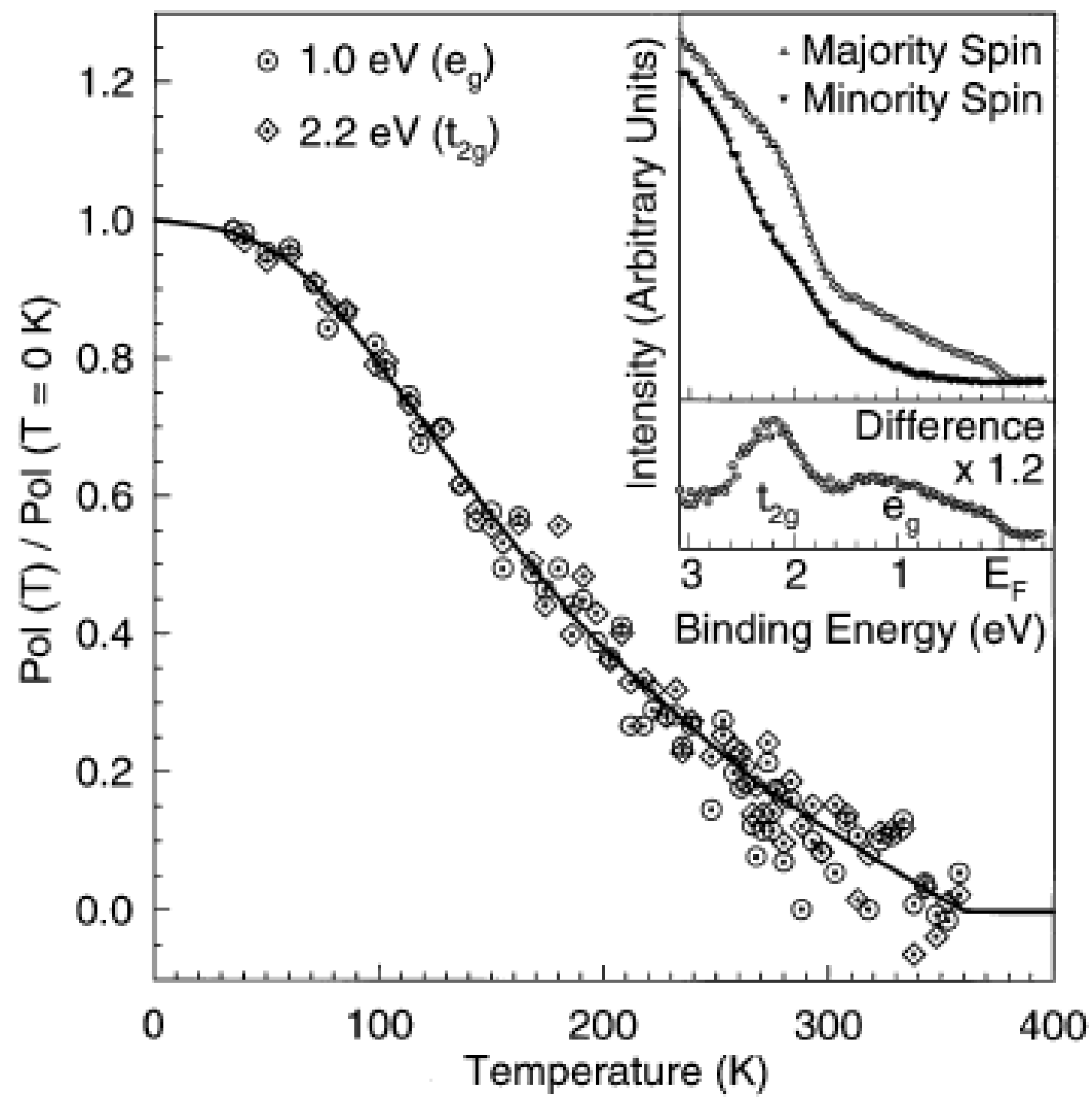

Figure 3 







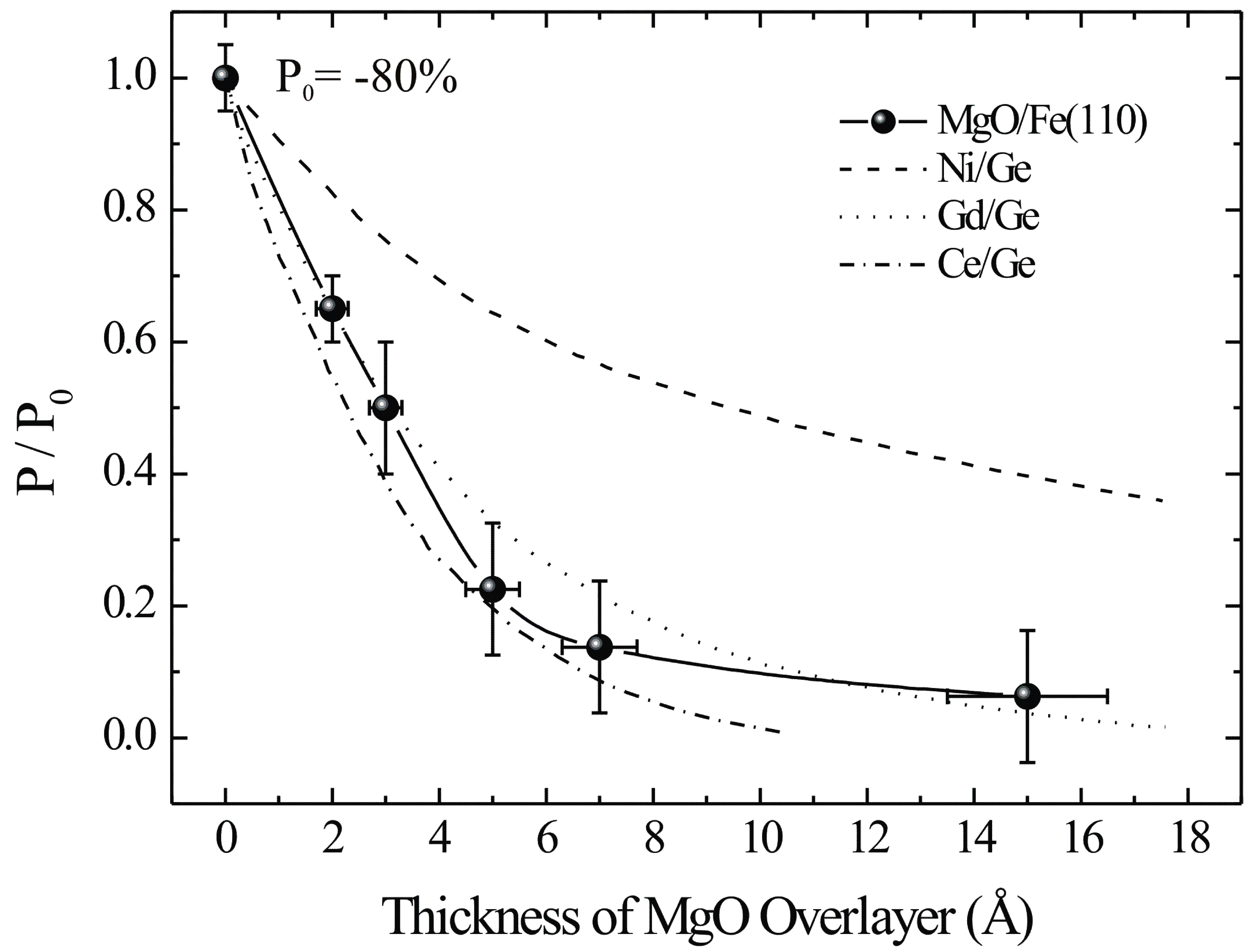






